\title{
Optimal control of vehicle dynamics for the prevention of road departure on curved roads
}

\author{
Yangyan $\mathrm{Gao}^{\mathrm{a} *}$, Timothy Gordon ${ }^{\mathrm{a}}$
}

\begin{abstract}
Run-off-Road crashes are often associated with excessive speed in curves, which may happen when a driver is distracted or fails to compensate for reduced surface friction. This work introduces an Automated Emergency Cornering (AEC) system to protect against the major effects of over-speeding on curves, especially lateral deviation leading to lane or road departure. The AEC architecture has two levels: an upper level to perform motion planning, based on the optimal control of a nonlinear particle model, and a lower level to distribute the resulting two-dimensional acceleration reference to the available actuators. The lower level adopts the recently introduced Modified Hamiltonian Algorithm (MHA), which continuously adjusts the priority between mass-centre acceleration and yaw moment demands derived from lateral stability targets. AEC makes use of a high precision map and triggers control interventions based on vehicle kinematic states and detailed road geometry. To avoid false-positive interventions, AEC is triggered only when excessive road departure is predicted for the optimal particle motion. AEC then takes control of steering and individual wheel brake actuators to perform autonomous motion control for speed and path curvature at the limits of available friction. The AEC system is tested and evaluated using the high-fidelity simulation software CarMaker.
\end{abstract}

Index Terms - Vehicle Dynamics and Control, Autonomous Vehicles, Active Safety, Optimal Control, Collision Avoidance, Lane Departure Prevention.

\section{INTRODUCTION}

Single-vehicle roadway departure crashes account for around $20 \%$ of all police-reported crashes in the USA [1]. Approximately one third of such crashes occur during a turn and nearly half of those crashes involved excessive speed [2], [3]. Furthermore, run-off-road (ROR) crashes are more likely to occur in adverse weather conditions, indicating that vehicle friction limits are an important factor. The ability to control speed and path on curves also depends on driver skill and decision-making. As noted in [1], the mechanisms behind ROR crashes are often complex, involving a combination of factors: driver performance, speed, path and lateral stability, together with road surface friction. There remains a challenge in vehicle system dynamics to address this integrated control problem, supporting the design of future advanced driver assistance systems (ADAS) to comprehensively reduce the risk of ROR crashes on curved roads.

Existing implementations of ADAS to protect against ROR and related lane departure crashes have typically suffered from problems of false positives. In [4], results from a Field Operational Test (FOT) of a Curve Speed Warning (CSW) system exhibited excessive numbers of false alarms. And in

\footnotetext{
${ }^{*}$ Corresponding author, ${ }^{a}$ School of Engineering, University of Lincoln, Brayford Pool, Lincoln LN6 7TS, UK; Email: yagao@lincoln.ac.uk
}

the case of true positive alarms, successful intervention relies on the prompt and skillful action of the driver. To avoid this limitation, automatic control is preferable to simple driver warning, but the problem of false positives remains to be solved.

In this paper we propose the concept of Automated Emergency Cornering (AEC). AEC is conceived to be analogous to Autonomous Emergency Braking (AEB), a system that automatically applies braking when the vehicle is about to suffer a frontal collision [5]. AEB aims to prevent or mitigate an impending collision if the driver fails to intervene, and does so at the last possible moment. Recent statistics show that AEB is effective in reducing the number and severity of these crashes [6]. Hence, by sharing the same general design concept, AEC can be expected to show similar benefits.

To achieve this requires a sophisticated chassis control system for the simultaneous control of path, speed and stability, with the capability of operating at or close to the limits of tyre adhesion. Not only this; it requires a predictive capability to engage AEC at the moment when further delay inevitably leads to excessive road or lane departure, even if the human driver is skilled and responsive.

ADAS control to prevent ROR crashes has been proposed previously, see for example [7], [8], [9], [10], [11], [12], [13], [14], [15], [16]. In existing literature, the common approach is to assume an incompetent driver and provide warning, overriding control or shared control to trigger a crash-avoiding intervention. For example, in [15], a fuzzy sliding mode control (FSMC) method is proposed based on visual preview distance to address the shared control for road departure prevention. The system provides support for the driver, but in a way that can interfere with normal driving, raising issues of acceptability and unintended interactions.

Many approaches have a practical bias, e.g. in [8] a road departure prevention system for heavy trucks was proposed based on autonomous steering with a motion reference obtained from vehicle localization relative to a digital map. The system would detect lane or road departure based on current position, then perform path corrections and (according to the design concept) speed control, applied to bring the vehicle to a safe condition using full autonomous control. The aim is to protect (especially) a drowsy driver who loses the ability to adequately control the vehicle.

In [13] a more formal approach was adopted using local kinematics and road geometry to derive reachability sets to predict road departure. However, in this work, only linear vehicle dynamics and constant curvature roads were considered, and no real-time controller was presented. 
Indeed, all the above systems can be considered 'soft', in the sense that the limits of friction are avoided; but friction limits ultimately define the threshold for when road departure becomes inevitable.

An existing 'hard' system, working at the limits if friction, is Electronic Stability Control (ESC). While ESC has proven safety benefits [17], the effect of road departure accidents is primarily through overcoming loss of control. ESC helps the driver maintain lateral stability, but is not equipped with environmental sensors and cannot directly prevent road departure. Hence it is hardly ever considered in the context of road departure prevention.

A number of control techniques have been proposed for motion control at or near the limits of tyre adhesion [18], [19], [20], [21], [22], [23], [24], [25], [26], [27], [28], [29], [30]. Many use a 'black box' approach, based on model predictive control (MPC), especially linear-time varying MPC (LTVMPC) or via nonlinear programming (NMPC); in such cases constrained numerical optimization operates within the control loop. In [19] NMPC was used for path following in a general framework; a hierarchical controller was used, the upper level defining an optimized path, the lower level tracking that path. In [20], feedback linearization was used to improve handling, in response to driver commands, up to the limits of friction. The approach included parameter adaptation and again used nonlinear programming. In [26] NMPC was again used to improve lateral stability in a general way, using driver inputs to derive a path reference. LTV-MPC is also commonly used; it has the advantage of reducing the complexity and challenge of optimization, while accounting once more for friction limits, e.g. [18], [29]. LTV-MPC has been applied to minimum time cornering, collision avoidance etc.

While the above papers describe general control approaches using MPC, some progress has been made in applying these techniques to emergency scenarios similar to that of AEC. In [31] it is noted that, in emergency scenarios, lateral stability should sometimes play a secondary role to speed and path control. MPC was used to track a nominal path and follow a desired speed profile. For the AEC application, there remains the difficulty of computing the target path in real-time; speedpath planning becomes highly coupled and also dependent on the low level chassis control performance.

Also, as is typical with MPC, there are a large number of weighting parameters to be pre-tuned for the online optimization. In the work of [32] some aspects of the preview control are similar to those required for AEC, using look-ahead to determine safe cornering speeds based on CG (particle) motion. However, again there is no attention to integration of speed planning with lateral dynamics, an important element of AEC control.

While 'black box' MPC techniques can provide effective control up to the limits of tyre friction, and can be applied to road departure prevention, there remain a number of limitations, in addition to those mentioned above. In particular, the necessary constrained optimization is not guaranteed to converge, and the iteration time is not fixed. Also, since the internal working of the controller is hidden, low-level control diagnostics are not easily available. Hence, to protect against algorithmic failure, a completely redundant back-up controller would be required.

As an alternative to real-time numerical optimization, explicit nonlinear control has been proposed based on simplifying chassis control concepts. In [30] a simple but effective control law for steering was used to track a desired body side-slip angle derived from a primary target of controlling the acceleration vector at the mass centre (CG). The approach of [30] is similar to that of [25] which also adopts a CG acceleration target based on a lower-level body sideslip controller. A similar approach is also presented in [27], where a simple nonlinear controller was implemented, using yaw rate as a synthetic control input. These papers focus is primarily on lateral dynamics, and the AEC concept relies on the highly coordinated control of both speed and path curvature at the upper level.

In the above literature, no approach offer all of the characteristics desired for AEC:

- explicit control structure

- explicit consideration of friction limits

- combined path and speed reference

- predictive capability for future off-tracking, with nearoptimal performance

- attention to current states and road boundaries for updating the reference.

Regarding the final item, this includes the unnecessary separation of control loops for speed and curvature.

Hence, in this work, we follow the methodology of the Modified Hamiltonian Algorithm (MHA) [24], [33], [34], [28] which potentially enjoys all of these attributes, without introducing any high degree of complexity or iteration within the control loop. As is common, we adopt a twolevel hierarchical controller, the upper level defining a motion reference, the lower level allocating commands to steering and individual wheel brake actuators. The motion reference is based on a friction-limited particle in the form of a target CG acceleration vector. The lower level (MHA) controller commands yaw motion as well as CG force targets, in a form that is easily allocated to the actuators. Details are provided in the following.

The paper is structured as follows: Section II defines the dynamic system, including the road (track) definition. Section III describes the lower-level MHA chassis control system and Section IV defines the upper-level particle reference for AEC. Section V-A presents simulation results in CarMaker, and Section VI provides conclusions.

\section{System DESCRIPTION}

The overall dynamic system is represented in Figure 1, comprising vehicle, driver (model), environment and safety technology. Reduced-order vehicle models are also used for tyre calibration and internally within the AEC controller.

\section{A. Vehicle Models}

Vehicle simulation uses IPG CarMaker, a high-fidelity vehicle dynamics simulation software widely employed in the automotive industry [35]. It includes a library of representative 


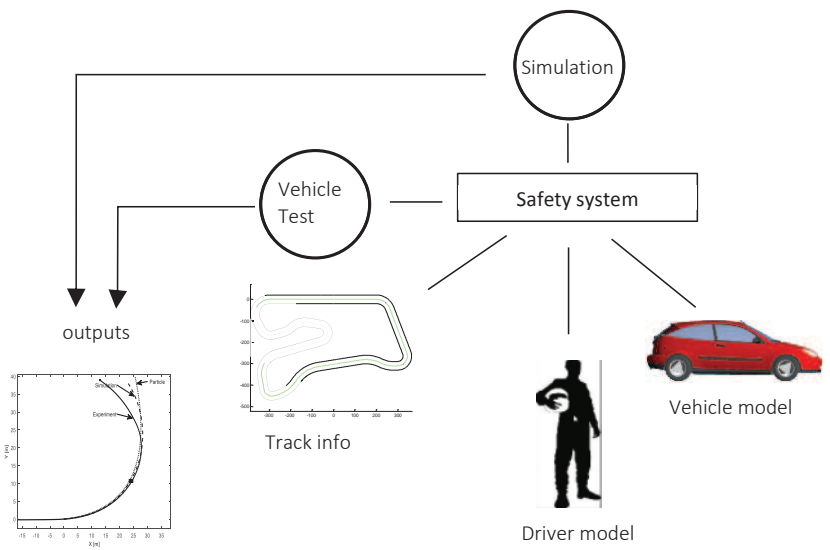

Fig. 1. Schematic of the overall system.

vehicle models, and here we select the 'Demo Ford Focus' model, similar to a test vehicle used for earlier validation of the MHA controller [28]. Included is the 'Real-Time (RT)' tyre model with data file 'DT_195_65R15.tdx' [35]. The major parameters are given in Appendix B.

The particle model, mentioned above, is formalized in Section II-C below. A further vehicle model is used in the form of a two-track planar model with seven mechanical degrees of freedom, three for body motion and four for wheel rotation. The model is used as a reference model to develop the integrated chassis controller in Section III. The equations of motion are as follows:

$$
\begin{aligned}
& m\left(\dot{v}_{x}-v_{y} \cdot \dot{\psi}\right)=F_{x}-F_{x}^{e} \\
& m\left(\dot{v}_{y}+v_{x} \cdot \dot{\psi}\right)=F_{y}-F_{y}^{e} \\
& I_{z z} \ddot{\psi}=M_{z} \\
& \dot{\omega}_{i}=I_{\mathrm{w}}^{-1}\left(T_{i}-R_{\mathrm{w}} F_{x i}^{t}\right) .
\end{aligned}
$$

Here $\left(F_{x i}, F_{y i}\right)$ are respectively the longitudinal and lateral components of the resultant forces from the tyres, resolved in body-fixed coordinates. $M_{z}$ is the yaw moment arising from the same tyre forces, and $\left(F_{x}^{e}, F_{y}^{e}\right)$ are external body forces e.g. arising from aerodynamic drag. The fourth equation determines the wheel rotational dynamics, controlled by the applied torque $T_{i}$ at each wheel, as well as the reaction force $F_{x i}^{t}$ at the contact patch. Here $R_{w}$ is the wheel radius and the wheel locations are labelled $i=(1,2,3,4)$ corresponding to (front-left, front-right, rear-left, rear-right) respectively.

The 7-DOF model includes a tyre model, which is used within the MHA controller [24]. It is a load-dependent combined-slip model based on the Pacejka magic formula (MF) [36]:

$$
P(x)=D \sin \left(C \tan ^{-1}\left(B x-E\left(B x-\tan ^{-1} B x\right)\right)\right)
$$

The model uses a normalized slip vector and a common shape function, with anisotropic scaling in the longitudinal and lateral directions - see [37], [38], [39] for further details.

\section{B. Tyre Model Fitting}

It is a deliberate choice that the controller's internal (MF) tyre model differs from that of the simulation model, since in real applications we cannot guarantee an accurate match to the tyres of an actual vehicle. However, to avoid excessive errors, some matching of the MF model to the simulation model is necessary. In this work, the 'real-time' (RT) tyre model RT_195_65R15 [40] is selected for simulation. The MF parameters $B, C, D$ and $E$ in Eqn. 2 are fitted for pure longitudinal slip, using nonlinear least-squares optimization at a single representative load. Then parameters $B, D$ are refitted for lower and higher loads, and the results put into a lookup table. For simplicity, and to avoid over-fitting, the shape parameters $C, E$ are held constant. Further fitting of $B, D$ is carried out for the lateral forces, and for combinedslip conditions a simple nonlinear interpolation procedure is followed [37], [38], [39].

Results are shown in Figures 2 and 3. It is seen that the simple model matches well in simple conditions, though without the complexity of the full RT_195_65R15 model.

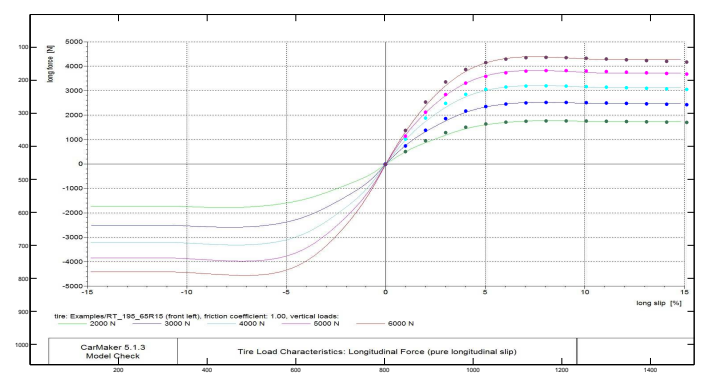

Fig. 2. Longitudinal tyre force fitting with various loads. Curves represent the RT_195_65R15 model, overlaid points are for the simpler MF model.

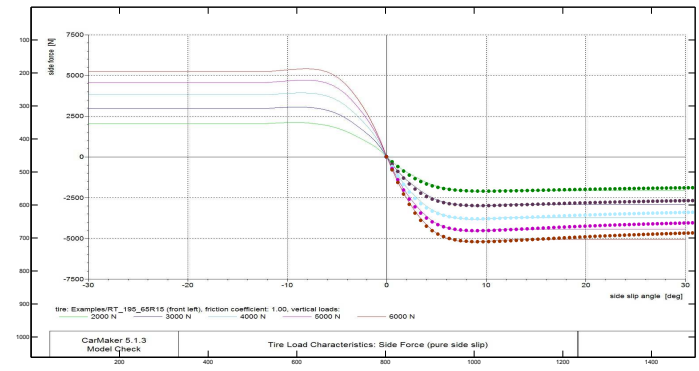

Fig. 3. Lateral tyre force fitting with various loads. Curves represent the RT_195_65R15 model, overlaid points are for the simpler MF model.

\section{Friction-limited Particle Model}

Friction limits at the tyres result in physical constraints on the mass-centre acceleration. Figure 4 shows an example, with the CarMaker vehicle model driven near the limits of friction on the Hockenheim racing circuit. When considering friction limits only, the boundary is approximately circular. While not an accurate representation of the vehicle dynamics, a circular CG acceleration bound provides a simple reducedorder model:

$$
\begin{aligned}
& \ddot{x}=u_{x} \\
& \ddot{y}=u_{y}
\end{aligned}
$$


Here $\left[u_{x}, u_{y}\right]^{T}$ is the control vector, freely chosen except for the constraint:

$$
\sqrt{u_{x}^{2}+u_{y}^{2}} \leq \mu_{p} g
$$

where from Figure 4 we estimate $\mu_{p} \approx 0.8$.

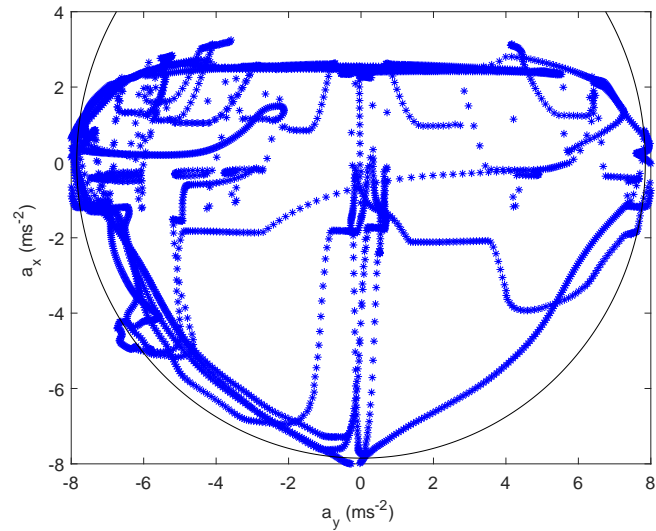

Fig. 4. G-G diagram for the CarMaker vehicle model driven on the Hockenheim circuit. Mass center accelerations are limited by a number of factors - tyre friction, driver actions and engine power. A nominal friction circle is added with $\mu_{p}=0.8$.

There are three related friction coefficients used in this paper: $\left(\mu_{s}, \mu_{p}, \mu_{c}\right)$ are respectively the surface friction in the $\mathrm{CM}$ vehicle model, the estimated particle-model friction limit used for chassis control and the particle friction limit used in the higher level AEC reference/ trigger algorithm (Section IV). The corresponding default values $(1.0,0.8,0.8)$ are used in this paper. The difference $\mu_{p}<\mu_{s}$ is expected, since tyre and suspension mechanics reduce the overall available vehicle acceleration. Alternative values are used in Section V-B where a reduced-friction scenario is considered.

\section{Road Definition}

Road information is required by the controller, and for preanalysis to compute a speed reference. Hence, in parallel to the internal CarMaker representation, road geometry is defined via a standardized $N \times 8$ track definition matrix $M$, with each row of the form

$$
M_{i}=\left[s_{x i}, x_{i}, y_{i}, t_{x i}, t_{y i}, n_{x i}, n_{y i}, c_{i}\right]
$$

The 'track' centre-line comprises a series of segments, each an arc of constant curvature $c_{i}=R_{i}^{-1}$. The $i^{\text {th }}$ arc starts at node $\left(x_{i}, y_{i}\right)$ in fixed inertial cartesian coordinates, and $\mathbf{t}_{i}=\left[t_{x i}, t_{y i}\right]^{T}, \mathbf{n}_{i}=\left[n_{x i}, n_{y i}\right]^{T}$ are respectively the track tangent and normal at node $i$ - see Figure 5. The first component, $s_{x i}$, is the cumulative arc length from the start of the track to the $i^{\text {th }}$ node. Any intermediate point $P$ on the track centre-line is defined by the continuous arc-length $s=s_{x}$, and the 8 components corresponding to $M_{i}$ are determined by interpolation relative to arc-length. A more general location for $P$ is represented by its track coordinates $P\left(s_{x}, s_{y}\right), s_{y}$ being the lateral offset to the left of the centre-line, parallel to the track normal $\mathbf{n}\left(s_{x}\right)$. The mapping between cartesian and track coordinates is well-defined and invertible in a wide region surrounding the centre-line. Note that the model assumes a horizontal flat surface, and when motion takes place within a single lane, the track centre-line coincides with the centre of the lane.

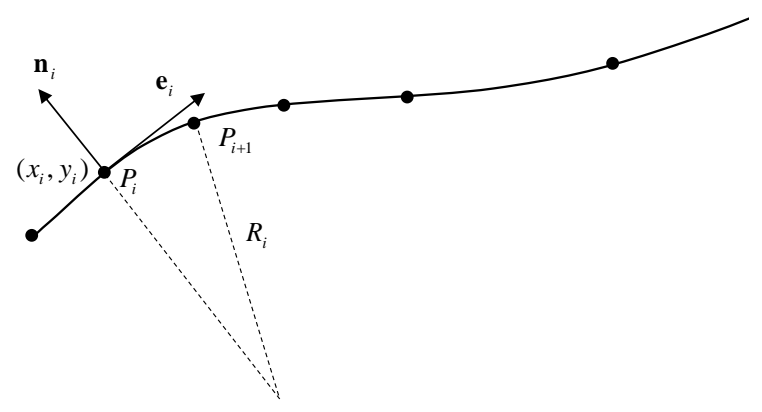

Fig. 5. Track model based on arcs of constant curvature. Each node corresponds to one row of the track definition matrix, Eqn. 5.

\section{E. Driver Model}

CarMaker includes a driver model, 'IPG Driver', capable of both speed and steering control [40]. We replace the speed controller using a custom PID controller, implemented in Simulink software, while retaining the IPG Driver steering model. The speed controller is to allow over-speeding at the entry to highway curves, and uses a reference speed $v_{\text {lim }}(s)$, defined as the maximum speed of the particle model when following the track centre-line. The limiting speed $v_{\text {lim }}(s)$ is determined as follows. Assuming the maximum available acceleration magnitude $\mu_{p} g, v(s)=v_{l i m}(s)>0$ is the solution of the following nonlinear differential equation:

$$
v(s)^{2} v^{\prime}(s)^{2}+c(s)^{2} v(s)^{4}=\mu_{p}^{2} g^{2}
$$

where the sign of $v^{\prime}(s)$ is chosen to maximise $v(s)>0$. On a circular track of constant curvature $c, v_{\text {lim }}=\sqrt{\mu_{p} g / c}$ is constant too, but more generally $v_{l i m}=v_{l i m}(s)$ is positiondependent, accounting for both lateral and longitudinal accelerations.

Figure 6 shows the speed profile for the Hockenheim racing circuit (see also Figures 14, 15 below). The reference speed, derived from Eqn. 6, is shown as the red solid line. It can be seen that the vehicle speed (blue dashed line) tracks the speed reference (red solid line) well, except where limited engine power causes under-speed. A nominal 'top speed' has been included, $v_{\max }=30 \mathrm{~ms}^{-1}$. Introducing a time delay for accelerator and braking actions, modelled as a transportation lag $\tau=0.5 \mathrm{~s}$, leads to over-speeding on curves (black dotted line). Hence, with delayed braking, the combined driver model will approach a tightening curve too fast and the vehicle will depart the road, providing test cases for AEC intervention.

\section{Chassis Control System}

During AEC operation, the particle model provides a reference $\mathbf{a}^{d}=\left[a_{x}^{d}, a_{y}^{d}\right]^{T}$ for the vehicle CG acceleration. The two-track model is then used to map this to force and moment 


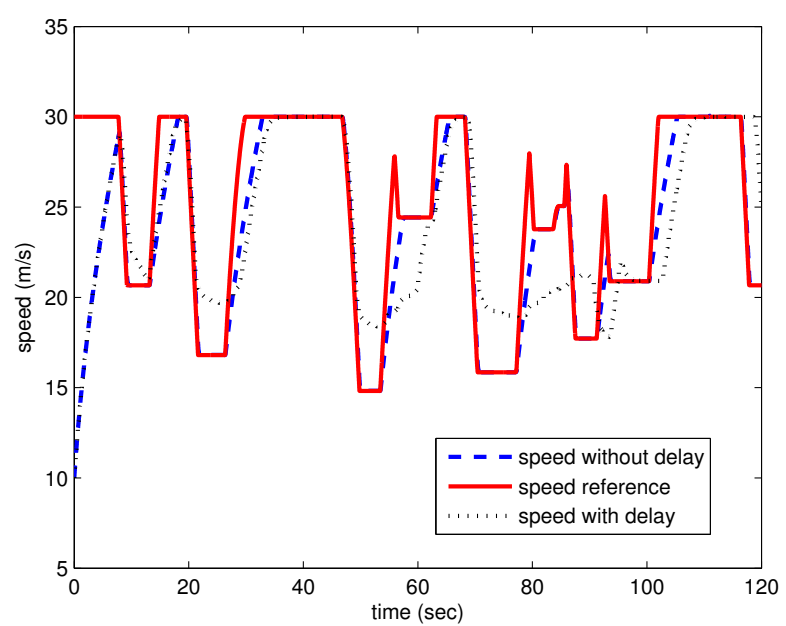

Fig. 6. Speed profile on the Hockenheim racing circuit. The reference speed $v_{l i m}\left(s_{x}\right)$ is based on the friction-limited particle with $\mu_{p}=0.8$. CarMaker simulations are used for the actual speeds. The black dotted curve shows the effect of an additional time delay in the speed controller.

requirements at the vehicle and actuator levels. The method has similarities with control allocation [41], [42], [43] where vehicle-level forces and moments $V$, the virtual controls, are used as an intermediate-level target for chassis actuator control.

\section{A. Optimal Control Formulation}

Firstly, an optimal control problem is formulated in terms of the virtual controls. The dynamic equations for the body motion in the 7-dof model have the simple form (Section II-A)

$$
\dot{x}=f(x)+G V
$$

where $x=\left[v_{x}, v_{y}, \psi\right]^{T}$ are the body motion variables, and $V=\left[F_{x}, F_{y}, M_{z}\right]^{T}$ are the body forces and moments. Matrix $G$ is the inverse inertia matrix, $G=\operatorname{diag}\left(m^{-1}, m^{-1}, I_{z z}^{-1}\right)$.

In principle, optimal control theory can be used to determine the virtual controls $V^{*}(t)$ [44], [45]. A cost function takes the form

$$
J=\Phi\left(x_{f}, t_{f}\right)+\int_{0}^{t_{f}} L(x, V) d t
$$

where $\Phi$ penalizes the terminal states and $L(x, V)$ penalizes deviations in the state trajectory and also the control effort. Because the focus here is on friction-limited control, it is not necessary to penalize control effort, so $L=L(x)$. In place of the penalty on control effort, friction constraints limit the virtual controls (also the actuator controls) to feasible limits.

According to optimal control theory [44], [45], the optimizing control $V^{*}(t)$ is found by minimizing the Hamiltonian function at each time instant

$$
H=p \cdot(f(x)+G V)+L(x)
$$

where $p(t)$ are the costate variables, which satisfy the adjoint equations:

$$
\dot{p}=-\frac{\partial H}{\partial x} \quad, \quad p\left(t_{f}\right)=\frac{\partial \Phi}{\partial x_{f}}
$$

As is well known, solving the combined state and costate equations, Eqn. 7, 10 (a two-point boundary value problem) is computationally intensive, and requires iterative numerical methods. Methods are not guaranteed to converge to a global minimum, and it is not feasible to directly implement this approach for AEC.

A simplification to the above was developed for post-impact vehicle motion control [21], [23]. The method, quasi-linearoptimal-control, determines the costate variables by solving the costate equations approximately, using linearization around a feasible (i.e. representative but sub-optimal) solution to the state equations. The method takes advantage of the simple form of the Hamiltonian, which is linear with respect to $V$ :

$$
H=m^{-1} p_{1} F_{x}+m^{-1} p_{2} F_{y}+I_{z z}^{-1} p_{3} M_{z}+H_{1}(x) .
$$

where $H_{1}(x)$ combines the terms independent of $V$. After the costate vector $\left[p_{1}, p_{2}, p_{3}\right]^{T}$ is determined, the actuator commands are found by minimizing $H$ at the instant in question. The costate equations should be solved at each time step. While computationally feasible, and without iterative optimization [21], [23], the method uses multiple simulations within the control loop and a more direct implementation is desirable.

The Modified Hamiltonian Algorithm, first introduced by the authors in [24], is formulated with emphasis on the physical interpretation of the costate functions, rather than their formal mathematical definition. Because only the control terms in Eqn. 11 are relevant to the minimization, and because the optimal control is independent of the term $H_{1}$, and also of any overall scale factor, we define the modified Hamiltonian function

$$
H=p_{x} F_{x}+p_{y} F_{y}+\lambda M_{z}
$$

where $\mathbf{p}=\left[p_{x}, p_{y}\right]^{T}$ is chosen as a unit-vector, reducing the number of unknowns from three to two.

Temporarily ignoring the term in $M_{z}$, it is clear that minimizing $H$ subject to a circular friction constraint implies $\left[F_{x}, F_{y}\right]^{T}=-\mu_{p} g \mathbf{p}$. Hence, the first assumption of MHA control is that $\mathbf{p}$ is selected to oppose the target acceleration vector,

$$
\mathbf{p}=-\mathbf{a}^{d} /\left|\mathbf{a}^{d}\right|
$$

The remaining unknown, $\lambda$, acts as a Lagrange multiplier associated with the yaw moment $M_{z}$. MHA adapts the value of $\lambda$ based on yaw moment demands. This is now considered in detail.

\section{B. Control Allocation via the Hamiltonian Function}

For yaw-sideslip control, $\lambda$ is adapted to track a desired yaw moment $M_{z}^{d}$ according to the rule:

$$
\lambda \rightarrow \lambda+S \cdot \sigma\left(\Delta M_{z}\right)
$$

where $S$ is a saturation limit, $\Delta M_{z}=M_{z}-M_{z}^{d}$ is a yaw moment error, $M_{z}$ is the estimated yaw moment from the controller (including contributions from longitudinal and lateral tyre forces) and $M_{z}^{d}$ is the desired yaw moment defined below. Function $\sigma$ is linear with unit saturation:

$$
\sigma\left(\Delta M_{z}, B\right)=\left\{\begin{array}{lc}
B \Delta M_{z} & \text { if }\left|B \Delta M_{z}\right|<1 \\
\operatorname{sgn}\left(\Delta M_{z}\right) & \text { otherwise }
\end{array}\right.
$$


Here we set $S=0.1, B=10^{-4}$. See [28] for details of how these parameters are chosen.

From Eqn. 12, the Hamiltonian can be expressed as a linear function of the individual tyre forces $\left(F_{x i}, F_{y i}\right)$ :

$$
\begin{array}{r}
F_{x}=\sum_{i} F_{x i} \\
F_{y}=\sum_{i} F_{y i} \\
M_{z}=\sum_{i}\left(x_{i} F_{y i}^{v}-y_{i} F_{x i}^{v}\right) .
\end{array}
$$

The superscript $v$ is used to indicate the vehicle coordinate system, with origin at the vehicle mass centre; $\left(x_{i}, y_{i}\right)$ are the fixed coordinates of the $i^{\text {th }}$ tyre contact patch with respect to vehicle axes.

Since the reference is defined in global coordinates $(g)$ and actuation takes place in local 'tyre' coordinate systems $(t)$, we perform transformations between these systems. The various rotational angles are shown in Figure 7. In particular we consider the general case of the $i^{\text {th }}$ wheel rotated through angle $\delta_{i}$ relative to the vehicle body, and later constrain the chassis to have parallel front steer, $\delta_{1}=\delta_{2}=\delta, \delta_{3}=\delta_{4}=0$. Using modified ISO coordinates, increases in yaw angle and steering angle are associated with increases in the various slip angles (body side-slip or tyre slip angle). This simplifies the sign convention for the equations that follow.

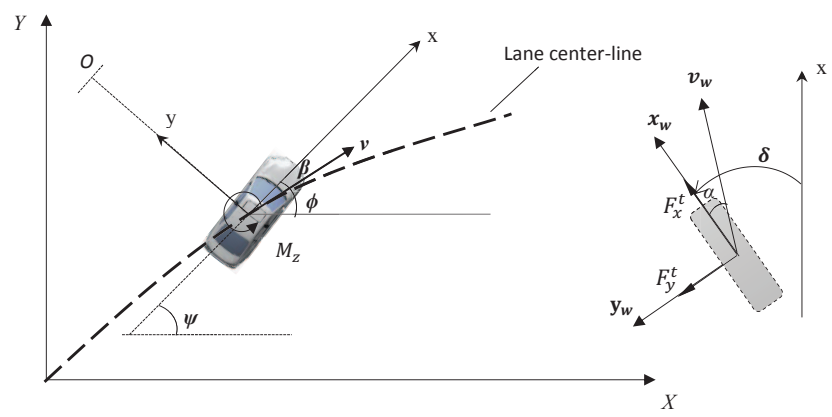

Fig. 7. Sign conventions for force, moment and wheel slip angle in the modified ISO coordinate system [46]. The left plot shows vehicle body sideslip angle $\beta$, path angle $\phi$ and yaw angle $\psi$. The right plot shows tyre slip angle and tyre forces.

From Figure 7, path angle $\phi$ is related to yaw angle $\psi$ and side-slip angle $\beta$ via the equation:

$$
\psi=\phi+\beta
$$

The (modified) Hamiltonian (Eqn. 12) is a scalar variable, invariant under coordinate transformations. $M_{z}$ is conveniently defined in vehicle body coordinates, so to aggregate the force terms we transform $\mathbf{p}$ from global to vehicle coordinates. Defining

$$
\boldsymbol{R}(\psi)=\left[\begin{array}{cc}
\cos \psi & -\sin \psi \\
\sin \psi & \cos \psi
\end{array}\right]
$$

it follows that

$$
\mathbf{p}^{v}=\boldsymbol{R}^{\mathrm{T}}(\psi) \cdot \mathbf{p}^{g}
$$

Now decompose $H$ into component wheel forces:

$$
H=\sum_{i} H_{i}
$$

where

$$
\begin{aligned}
H_{i} & =p_{x}^{v} F_{x i}^{v}+p_{y}^{v} F_{y i}^{v}+\lambda\left(x_{i} F_{y i}^{v}-y_{i} F_{x i}^{v}\right) \\
& =\left(p_{x}^{v}-\lambda y_{i}\right) F_{x i}^{v}+\left(p_{y}^{v}+\lambda x_{i}\right) F_{y i}^{v} \\
& =\tilde{p}_{x i}^{v} F_{x i}^{v}+\tilde{p}_{y i}^{v} F_{y i}^{v} \\
& =\tilde{\mathbf{p}}_{i}^{v} \cdot \mathbf{F}_{i}^{v}
\end{aligned}
$$

Here $\tilde{\mathbf{p}}_{i}^{v}$ includes the effect of the yaw moment and is local to the individual tyre. Again, the scalar product $H_{i}$ is invariant under rotation, and we rewrite $H_{i}$ in the individual tyre coordinate system via the rotation matrix $\boldsymbol{R}\left(\delta_{i}\right)$ :

$$
\begin{gathered}
\tilde{\mathbf{p}}_{i}^{t}=\boldsymbol{R}^{\mathrm{T}}\left(\delta_{i}\right) \cdot \tilde{\mathbf{p}}_{i}^{v} \cdot \\
H_{i}=\tilde{\mathbf{p}}_{i}^{t} \cdot \mathbf{F}_{i}^{t} \rightarrow \min
\end{gathered}
$$

and $\tilde{\mathbf{p}}_{i}^{t}$ are the linear tyre force coefficients of the 'local Hamiltonian' function $H_{i}$.

For online implementation this greatly simplifies the control algorithm, since the individual tyre forces are effectively decoupled. There is a minor interaction between the in-plane tyre forces and the vertical loads; but the effect is moderated by the suspension system and vertical loads can be estimated independently of the braking and cornering forces. Thus control integration between the individual wheels is determined by a single 'control integration' variable $\lambda$. While further actuator coordination is required due to constraints such as parallel front steering (see below) the simplicity of chassis control integration in this method is very clear.

\section{Control of Tyre Forces and Moments}

Figure 8 shows a typical tyre force map, each curve representing the forces available at a given slip angle due to changes in braking torque. Lines of constant $H_{i}$ are shown as green lines and $H_{i}$ is decreasing along the steepest descent direction shown. Stars show the points of minimum $H_{i}$ on any given curve.

For closed-loop control, we assume that brake forces can be rapidly modulated, so the star $(*)$ point can be rapidly obtained. For the current slip angle, the corresponding curve in Figure 8 determines the required value of $F_{x i}^{t}$ and hence the braking torque $T_{i}$. Here, for simplicity we assume $T_{i} \approx$ $F_{x}^{t} \cdot R_{\mathrm{w}}$, where $R_{\mathrm{w}}$ is the rolling radius of the wheel.

In slower time, the slip angle can be increased or decreased by (i) actively controlling the steer angle, or (ii) changing the body side-slip angle of the entire vehicle. The star with black circle shows an example of minimum Hamiltonian with the 'current' side-slip angle $\alpha=3.5^{\circ}$. Considering the neighbouring curves, $H_{i}$ decreases with slip angle, so for this wheel we should increase the steer angle or body side-slip angle to increase $\alpha$ if possible.

Taking a small perturbation around the 'star point', we obtain

$$
\frac{\partial H_{i}}{\partial \alpha_{i}} \approx \frac{H_{i}\left(\alpha_{i}+\varepsilon\right)-H_{i}\left(\alpha_{i}-\varepsilon\right)}{2 \varepsilon}
$$




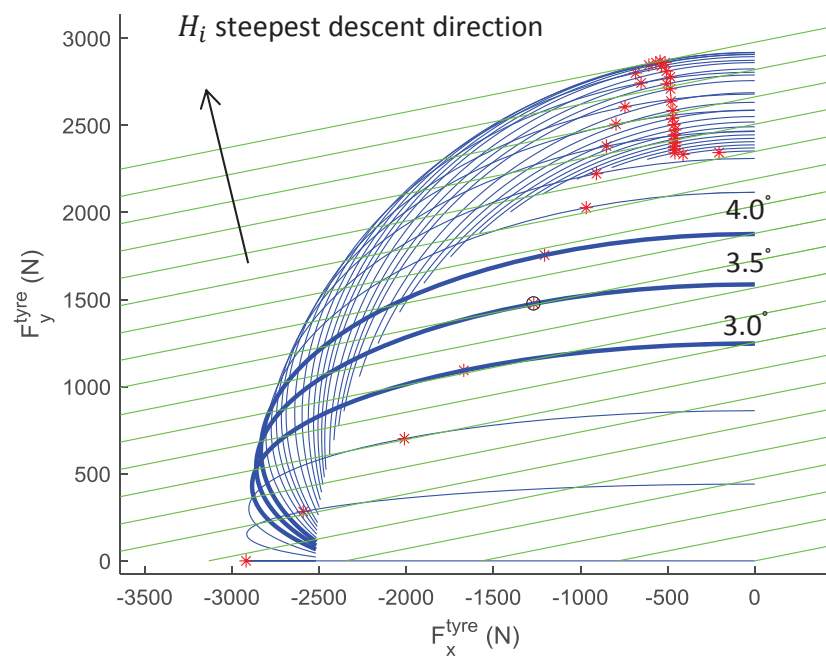

Fig. 8. Force map in tyre coordinates: each solid curve represents the range of forces available due to changes in braking torque for a given slip angle. Lines of constant $H_{i}$ are normal to the direction of steepest descent. Stars denote the points of minimum $H_{i}$ on each curve.

It is easy to show that when the vehicle side-slip angle $\beta$ is small,

$$
\alpha_{i}=\delta_{i}+\beta-v_{x}^{-1} \dot{\psi} x_{i}
$$

In the following we assume the following actuators: front axle steer with $\delta_{1}=\delta_{2}=\delta$ and the four independent wheel braking torques $T_{i}$. Coordination of slip angles at the front axle takes the form:

$$
\begin{aligned}
H_{\delta} & :=\frac{\partial H}{\partial \delta}=\frac{\partial H_{1}}{\partial \alpha_{1}} \cdot \frac{\partial \alpha_{1}}{\partial \delta}+\frac{\partial H_{2}}{\partial \alpha_{2}} \cdot \frac{\partial \alpha_{2}}{\partial \delta} \\
& =\frac{\partial H_{1}}{\partial \alpha_{1}}+\frac{\partial H_{2}}{\partial \alpha_{2}}
\end{aligned}
$$

Using Eqn. 26, assuming a steering rate limit $k_{\delta}$ for the front axle steering actuator [23], we locally reduce the value of $H$ via the control law

$$
\dot{\delta}= \begin{cases}-k_{\delta} \operatorname{sgn}\left(H_{\delta}\right) & \left|H_{\delta}\right|>\text { tol } \\ 0 & \text { otherwise }\end{cases}
$$

At the rear axle, with no steering actuator available, the body side-slip angle is used to modify $\alpha_{3}$ and $\alpha_{4}$. This requires yaw moment control and relates back to the desired yaw moment see Section III-D below. Body side-slip control is determined in a similar fashion, through the following Equation:

$$
H_{\beta} \equiv \frac{\partial H}{\partial \beta}=\sum_{i} \frac{\partial H_{i}}{\partial \alpha_{i}} \cdot \frac{\partial \alpha_{i}}{\partial \beta}=\sum_{i} \frac{\partial H_{i}}{\partial \alpha_{i}}
$$

Similar to Eqn. 29, we define a target side-slip rate based on the requirement to minimize $H$

$$
\dot{\beta}_{H}= \begin{cases}-k_{\beta} \operatorname{sgn}\left(H_{\beta}\right), & \text { if }\left|H_{\beta}\right|>t o l \\ 0, & \text { otherwise }\end{cases}
$$

Lateral stability limits are introduced to avoid increasing side-slip angle $\beta$ beyond some threshold value $\beta_{2}$, typically no more than around $8^{\circ}$. We introduce a saturation condition as follows

$$
\dot{\beta}^{d}= \begin{cases}-k_{\beta} \operatorname{sgn}(\dot{\beta}), & \text { if }|\beta|>\beta_{2} \\ 0, & \text { if }|\beta|>\beta_{1} \bigcap \beta \dot{\beta}_{H}>0 \\ \dot{\beta}_{H}, & \text { otherwise }\end{cases}
$$

where $0<\beta_{1}<\beta_{2}$. While the switching conditions are somewhat ad-hoc and require separate definition of threshold parameters $\left(k_{\beta}, \beta_{1}, \beta_{2}\right)$, each parameter has a direct meaning for controller design and such rules are typical of traditional control laws for body side-slip [47].

\section{Desired Yaw Moment}

To complete the MHA algorithm, we need to define the desired yaw moment $M_{z}^{d}$. From Eqn. 19 we determine the desired yaw rate

$$
\dot{\psi}^{d}=\dot{\phi}+\dot{\beta}^{d}
$$

Variable $\dot{\beta}^{d}$ is determined above and $\dot{\phi}$ is related to path curvature, which is found from the acceleration in path coordinates

$$
m v \dot{\phi}=m a_{y}^{p}=F_{y}^{g} \cos \phi-F_{x}^{g} \sin \phi
$$

$v$ being vehicle speed and $F^{g}$ being the force components determined from the tyre model above. Yaw moments are applied to track the desired yaw rate via a first order control law

$$
\tau \frac{d \dot{\psi}}{d t}=\dot{\psi}^{d}-\dot{\psi}
$$

Hence the desired yaw moment is

$$
M_{z}^{d}=\tau^{-1} I_{z z}\left(\dot{\psi}^{d}-\dot{\psi}\right)
$$

This completes the definition of the key MHA control equations.

In summary, Figure 9 shows a block diagram of the overall vehicle-chassis control system. The above equations describes the dashed rectangle block shown as the MHA control allocator. As mentioned previously, MHA performs both optimization and control allocation. Conventional control allocation computes the high level virtual controls as targets for actuator commands, and solves by further optimization [41], [42], [43]. By contrast, the modified Hamiltonian function is directly resolved into wheel-level components which are then individually minimized to determine actuator commands.

\section{AEC Optimality AND RefEREnCE ACCELERATION}

\section{A. Parabolic Path Reference (PPR)}

In earlier work, [22], [34], an optimal control intervention was determined for an over-speeding vehicle driving on a simple road geometry. There, the road comprised a straight segment followed by an arc of constant curvature $c_{0}$. The control objective was to minimize the maximum radial offtracking on the curve when the entry speed was higher than the limit imposed by friction and curvature - Figure 10. In 


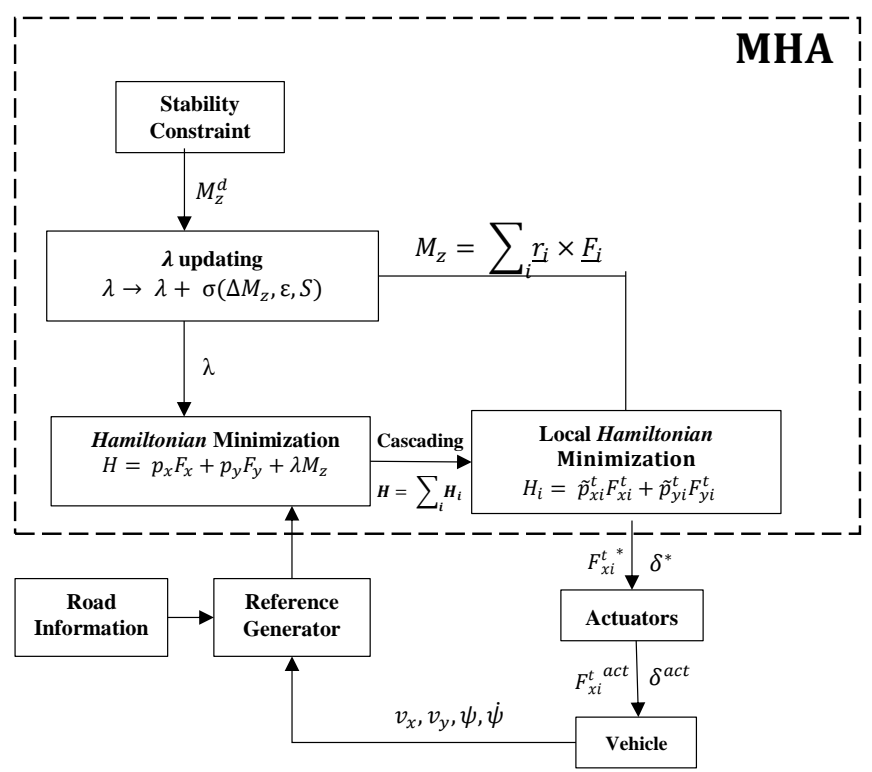

Fig. 9. Overall structure of the MHA algorithm. MHA updates the costate ratio $\lambda$ and uses linearity with respect to the virtual controls to distribute the vehicle-level control target to individual wheels.

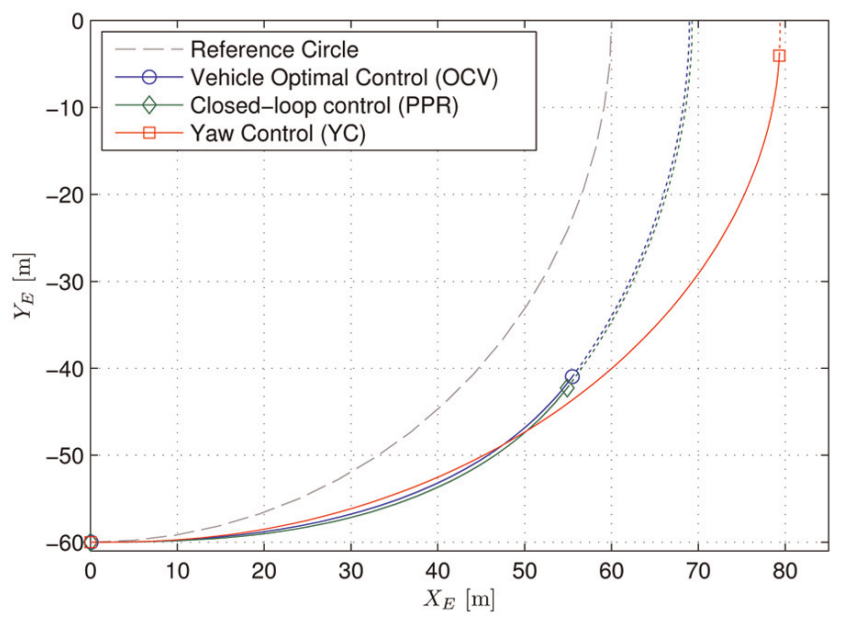

Fig. 10. Comparison of the optimal particle trajectory under PPR with the numerically optimized vehicle path (OCV) for a constant radius curve. [22]. Also shown is the vehicle path optimized through yaw moment control. Line markers indicate the point of maximum off-tracking.

this case it is not possible for a vehicle to follow the centreline of the road, which is shown as the 'reference circle'. The optimal vehicle CG motion closely matches a 'parabolic particle reference' (PPR), which is the motion of the frictionlimited particle model subject to an inertially fixed acceleration vector $\mathbf{a}^{*}$. In this simplified scenario, the initial velocity $\mathbf{v}_{0}$ is parallel to the track when intervention is triggered. The acceleration vector $\mathbf{a}^{*}$ is fixed in space at an angle $\theta^{*}$ behind the normal to the track at the trigger point:

$$
\cos \theta^{*}=\bar{v}^{-2}
$$

Here $\bar{v}=v_{0} / v_{l i m}$ and $v_{l i m}=\sqrt{\mu g / c_{0}}$ is the limiting speed for steady-state motion on the curved segment; we write $\mu_{p}=\mu_{c}=\mu$ here and in the remainder of this section. Also shown in Figure 10 is an ESC-like intervention derived from yaw moment optimization (labelled 'YC'). This performs less well, since speed reduction is not a control target for ESC [22]. Indeed, any alternative control strategy which directs the $\mathrm{CG}$ acceleration vector in a substantially different way, is guaranteed to result in poorer off-tracking performance [22] also see Theorem 4.1 below.

Real-world feasibility simply depends on being able to track the PPR reference acceleration while maintaining adequate control of yaw motion. This was demonstrated in [34], [28], where an instrumented vehicle, with manual steering and independently control wheel brakes, was able to track the PPR reference using the MHA algorithm of Section III, and hence achieve speed and off-tracking performance approaching that of the ideal particle.

\section{B. PPR for General Road Geometries}

The objective now is to generalise the previous analysis to a wider class of road geometries, especially to determine the target acceleration $\mathbf{a}^{*}$ when road curvature $c(s)$ is no longer constant. Further, AEC intervention should be triggered according maximum off-tracking demands, vehicle kinematics and road geometry.

Consider the case where a vehicle is approaching a sequence of positive curvature segments, with entry speed higher than $v_{\text {lim }}\left(s_{0}\right)$ of Eqn. 6, so off-tracking (to the right) becomes inevitable, see Figure 11. (Equally we could consider curvature to the right with positive off-tracking to the left). The current vehicle $\mathrm{CG}$ position, in track coordinates, is $S\left(s_{0}, d_{0}\right)$, and the CG velocity vector is $\mathbf{v}_{0}$.

The off-tracking velocity at a preview point $P\left(s_{0}+e, D\right)$ is defined by

$$
v_{\perp}(e)=-\mathbf{n}_{P} \cdot \mathbf{v}_{P}
$$

where $\mathbf{n}_{P}$ is the track-normal at $P$. The value of $v_{\perp}(e)$ depends on the motion control applied between $S$ and $P$, and - motivated by PPR - in the definition we assume the vehicle follows a fixed $\mathrm{CG}$ acceleration aligned with $\mathbf{n}_{P}$ :

$$
\mathbf{a}_{P}=\mu g \mathbf{n}_{P}
$$

We expect to find a preview point $e_{1}$ for which $v_{\perp}\left(e_{1}\right)>0$. In Figure 11, this already occurs at $S$, so a valid choice is $e_{1}=0$. More generally, we make the 'scenario assumption' that $v_{\perp}\left(e_{1}\right)>0$ for some $e_{1} \geq 0$.

While the road geometry is to be as general as possible, we assume it has curvature in just one direction, $c(s) \geq 0$ for $s \geq s_{0}+e_{1}$, at least up to some distant point on the track. It seem plausible to assume

$$
v_{\perp}\left(e_{2}\right)<0
$$

for some future point $e_{2}>e_{1}$, i.e. the CG acceleration of Eqn. 39 eventually reverses the sign of the off-tracking velocity. In that case, since $v_{\perp}(e)$ is a continuous function and its value changes sign, there must be a preview distance $e^{*}$ in the interval $\left(e_{1}, e_{2}\right)$ for which

$$
v_{\perp}\left(e^{*}\right)=0
$$



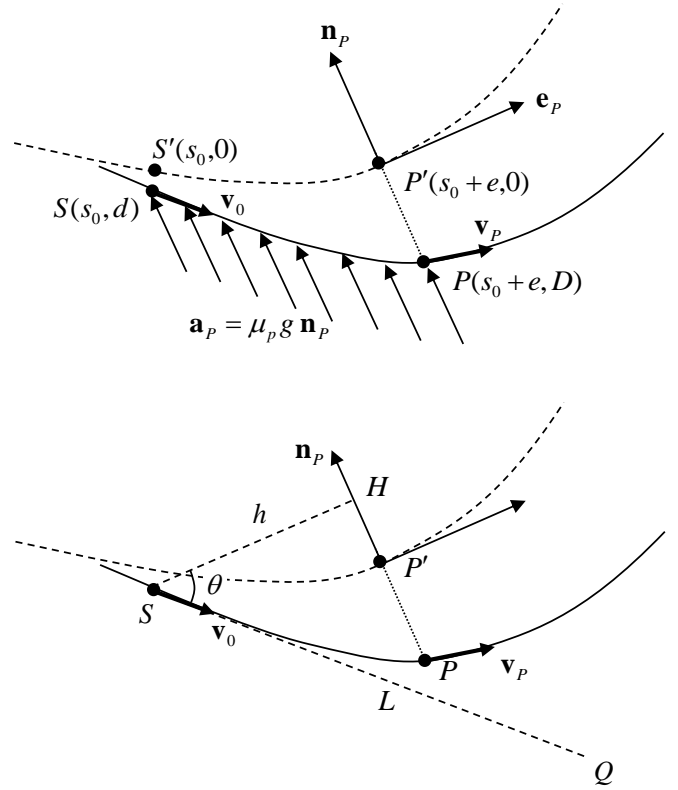

Fig. 11. Geometric definition of the previewed lateral velocity $v_{\perp}$. Parabolic motion occurs under constant acceleration $\mathbf{a}_{P}$. Unit vectors $\mathbf{e}_{P}, \mathbf{n}_{P}$ respectively define the track tangent and normal at the previewed point $P^{\prime}$ on the track centre-line (dashed curve). $h$ is the distance between $S$ and the track normal, with $S H$ drawn parallel to $\mathbf{e}_{P} \cdot v_{\perp}$ is the component of $\mathbf{v}_{P}$ in the direction $-\mathbf{n}_{P}$. Line $S Q$ represents an alternative motion under straight-line braking, and $L=|S Q|$ is the minimum stopping distance.

If more than one such point exists, the value of $e_{2}$ can be reduced so that $e^{*}$ is a unique solution.

The existence of $e_{2}$, and hence $e^{*}$ may seem obvious: a concerted path-lateral control effort will eventually reverse the off-tracking tendency. However, it seems important enough to provide a formal verification - Appendix A. Here the general reasoning is given. Referring to Figure 11, motion parallel to $\mathbf{e}_{P}$ is orthogonal to the acceleration vector and hence the velocity component is constant. Hence the time to reach $P$ from $S$ is found from distance $h$ :

$$
T_{P}=\frac{h}{v_{0} \cos \theta}
$$

Motion parallel to $\mathbf{n}_{P}$ has constant acceleration $\mu g$ and hence

$$
\begin{aligned}
v_{\perp} & =v_{0} \sin \theta-\mu g T_{P} \\
& =v_{0} \sin \theta-\frac{\mu g h}{v_{0} \cos \theta}
\end{aligned}
$$

Introducing the minimum straight-line stopping distance $L$, then $v_{0}^{2}=2 \mu g L$, and Eqn. 43 for the lateral velocity is rewritten:

$$
v_{\perp}=\frac{v_{0}}{2 \cos \theta} \cdot\left(\sin 2 \theta-\frac{h}{L}\right) .
$$

Hence, increasing the preview distance will typically cause $h$ in Figure 11 to exceed $L$, so the bracketed expression in Eqn. 44 becomes negative, showing that both $e_{2}$ and $e^{*}$ exist.

Writing $P^{*}$ for the vertex of the parabola associated with $e^{*}$ we propose the following inertially-fixed acceleration vector as the required PPR generalization:

$$
\mathbf{a}^{*}=\mu g \mathbf{n}^{*}
$$

where $\mathbf{n}^{*}=\mathbf{n}_{P^{*}}$ is the track normal at $P^{\prime *}$.

\section{PPR Optimality}

In [22], PPR optimality for the constant radius curve was derived using Pontryagin's Minimum Principle. Here, an alternative geometric analysis is provided.

Theorem 4.1 (PPR Optimality): Under the scenario assumptions above, control from $S$ under constant acceleration $\mathbf{a}^{*}$ is optimal for the friction-limited particle, i.e. for any other control strategy, maximum off-tracking is greater than for motion under $\mathbf{a}^{*}$.

Proof Introduce local coordinates $(\xi, \eta)$, as shown in Figure 12 , with origin at $S$ and axes aligned with the track normal and tangent unit vectors at $P^{\prime *}$. The candidate optimal control results in a path $\left(\xi_{1}(t), \eta_{1}(t)\right)$ from $S$ to $P^{*}$, which is the vertex of the parabola. Since $c(s) \geq 0$, it is sufficient to show that any other feasible control input has a trajectory $\left(\xi_{2}(t), \eta_{2}(t)\right)$ that penetrates the shaded region in the figure, for in that case off-tracking clearly exceeds distance $\left|P^{*} P^{* *}\right|$.

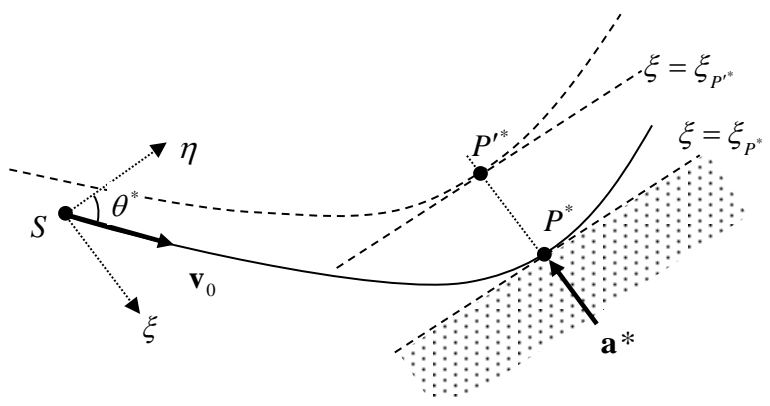

Fig. 12. Coordinates $(\xi, \eta)$ aligned to the track normal $\mathbf{n}^{*}$ and tangent $\mathbf{e}^{*}$ at $P^{\prime *}$. The optimizing acceleration vector $\mathbf{a}^{*}$ is parallel to $\mathbf{n}^{*} . P^{*}$ is the vertex of the optimizing parabola.

The candidate optimal trajectory satisfies the equations

$$
\ddot{\xi_{1}}=-\mu g \quad, \quad \ddot{\eta}_{1}=0
$$

with initial conditions (see Figure 12) $\xi_{1}(0)=0, \dot{\xi}_{1}(0)=$ $v_{0} \sin \theta^{*}, \eta_{1}(0)=0, \dot{\eta}_{1}(0)=v_{0} \cos \theta^{*}$. We set $t=0$ at point $S$, and write $t=T^{*}$ at the vertex.

From the friction constraint, Eqn. 4, any alternative trajectory $\xi_{2}(t)$ satisfies an equation of the form:

$$
\ddot{\xi}_{2}=-\mu g+\alpha(t)
$$

where $\alpha(t) \geq 0$ and $\alpha(t)>0$ for some finite period of time otherwise, from the constraint, $\ddot{\eta}_{2}=0$ and the same parabolic trajectory is followed.

The deviation in $\xi$ between the two trajectories is $\xi_{e}=$ $\left(\xi_{2}-\xi_{1}\right)$. Then

$$
\ddot{\xi}_{e}=\alpha(t) \geq 0
$$

with zero initial conditions. Integrating twice with respect to $t$ :

$$
\xi_{e}\left(T^{*}\right)=\int_{0}^{T^{*}} \int_{0}^{t^{\prime}} \alpha(t) d t d t^{\prime}>0
$$


Hence, at time $T^{*}, \xi_{2}>\xi_{P^{*}}$ and the alternative trajectory penetrates the shaded region. Hence off-tracking is greater than for the candidate optimal control.

Apex point. In Figure 12, $P^{*}$ determines where maximum off-tracking will occur, assuming an ideal control intervention. The off-tracking distance $D^{*}=\left|P^{*} P^{* *}\right|$ gives a best-case estimate of the maximum future off-tracking. This is valid and useful even if the AEC chassis controller cannot perfectly match the acceleration reference. The above theorem shows that maximum off-tracking is entirely dependent on the $\mathrm{CG}$ acceleration response in comparison to the reference particle. The vertex of the optimizing parabola, $P^{*}$, will be called the 'apex point'. Finding $P^{*}$ is crucial for the AEC trigger and control reference, and is found by searching an on-board digital map - see below.

The above generalizes the PPR analysis of [22], where in Figure 11 the track ahead of $s_{0}$ has constant curvature $c_{0}=$ $R_{0}^{-1}$ and intervention is triggered with $S$ on the track centreline (i.e. $d=0$ ); further, $\mathbf{v}_{0}$ is parallel to the track tangent. Simple geometry gives $h=R_{0} \sin \theta$, and from Eqn. 43, $v_{\perp}=0$ occurs when

$$
v_{0} \sin \theta=\frac{\mu g R_{0} \sin \theta}{v_{0} \cos \theta}
$$

and hence the optimizing angle is given by

$$
v_{0}^{2} \cos \theta^{*}=\mu g / c_{0}
$$

in agreement with Eqn. 37.

\section{AEC Trigger and Apex Search}

As discussed, AEC is to trigger when the best-case predicted off-tracking exceeds some prescribed threshold, $D^{*}>D_{0}$. In the following, an implementable algorithm is proposed for finding and evaluating the apex point $P^{*}$, and hence deciding if and when to trigger AEC.

First, an AEC event flag $f_{e}$ is introduced, to represent the following conditions:

$$
f_{e}= \begin{cases}+1, & \text { AEC function on, left turn } \\ -1, & \text { AEC function on, right turn } \\ 0, & \text { AEC function off. }\end{cases}
$$

During normal driving, $f_{e}=0$, and this 'off-state' is maintained until $v \geq v_{\text {lim }}$ is detected. In that case a function $F_{a e c}\left(\Gamma, f_{e}\right)$ is evaluated

$$
F_{a e c}:\left(\Gamma, f_{e}\right) \mapsto\left(P^{*}, \mathbf{a}^{*}, f_{e}^{\prime}\right)
$$

where $f_{e}^{\prime}$ is the updated event flag and $\Gamma$ denotes the geometric and kinematic information required at each time step by the AEC function. $\Gamma$ consists of the position and velocity states of the vehicle, together with a digital map giving the road information (geometry and friction level). If $P^{*}$ is found with $D^{*}>D_{0}$, then $f_{e}^{\prime}= \pm 1$ is returned, together with the acceleration reference $\mathbf{a}^{*}$. During the following, $F_{a e c}$ is evaluated at each time-step to give an updated $\mathbf{a}^{*}$. Finally, the AEC intervention finishes when the local lateral velocity $v_{\perp}(0)$ switches from positive to negative. The overall decision logic is given in Figure 13.

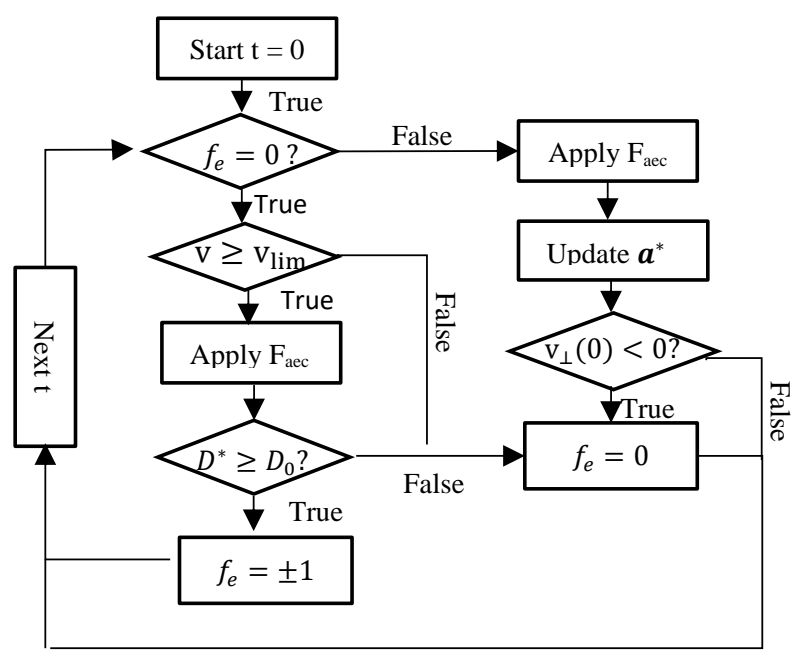

Fig. 13. Flow chart of the AEC reference / trigger system. Control is only applied when $f_{e}= \pm 1$, which requires the initial detection of an over-speed condition as well as continuous reporting of a valid detection. Intervention continues with updating of $\mathbf{a}^{*}$ (closed loop) until $v_{\perp}^{0}<0$ indicates the end of the intervention.

Function $F_{a e c}$ determines the initial choice of dominant road curvature using the position of point $Q$ in Figure 11, as determined from $\mathbf{v}_{0}$ and the straight-line braking distance. If the lateral coordinate is negative, $s_{y}(Q)<0$, the overall curvature is deemed positive. In general,

$$
f_{e}=-\operatorname{sgn}\left(s_{y}(Q)\right) .
$$

Next, $F_{a e c}$ performs a one-dimensional numerical search for the apex point, starting at the preview distance of $Q$,

$$
e_{0}=s_{x}(Q)-s_{0}
$$

If $v_{\perp}\left(e_{0}\right)>0$ the search is carried out forwards, with $e>e_{0}$; if $v_{\perp}\left(e_{0}\right)<0$ the search is backwards, with $e<e_{0}$. In either case, the apex point is found with $v_{\perp}\left(e^{*}\right)=0$ and hence $P^{*}$ and $\mathbf{a}^{*}$ are determined.

\section{Results}

\section{A. Hockenheim circuit}

Here the AEC system is tested in simulation, using the relatively complex geometry of the Hockenheim racing circuit. Figure 14 shows a satellite image and Figure 15 highlights a number of curves of interest. As mentioned in Section II-E, ROR risk was artificially created by including a time delay in the speed controller, so that braking into curves is delayed. Setting $D_{0}=0.8 \mathrm{~m}$ in the above algorithm, vehicle simulation was performed using CarMaker, both with and without AEC protection.

AEC was triggered on the five curves, labelled $A$ to $E$, and Figures 16, 17 show the vehicle path and $C G$ acceleration on two of these curves. Three acceleration vectors are shown in each plot, also indicating the times when AEC is active. These are: (a) the desired acceleration reference $\mathbf{a}^{d}=\mathbf{a}^{*}$ obtained from $F_{a e c}$ (red); (b) $\mathbf{a}^{c m d}$ predicted from 


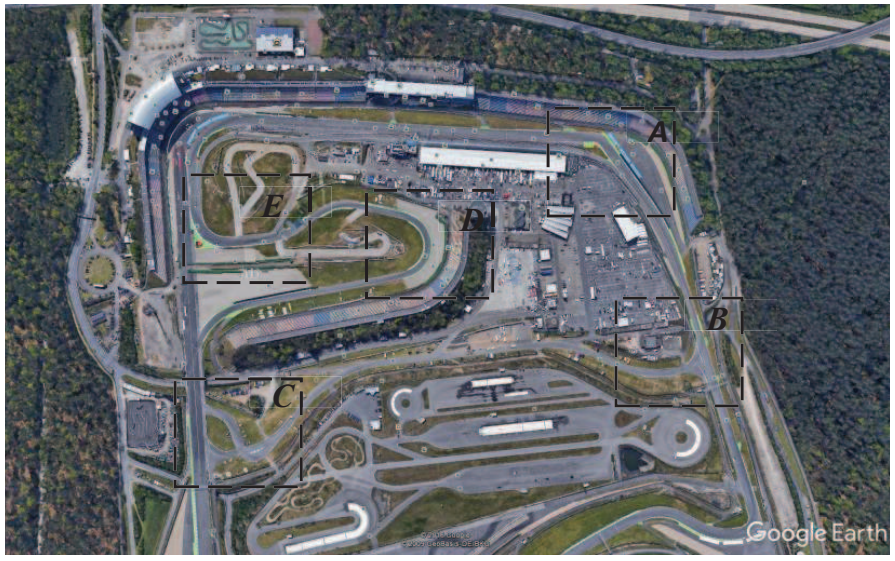

Fig. 14. Satellite image of the Hockenheim racing circuit.

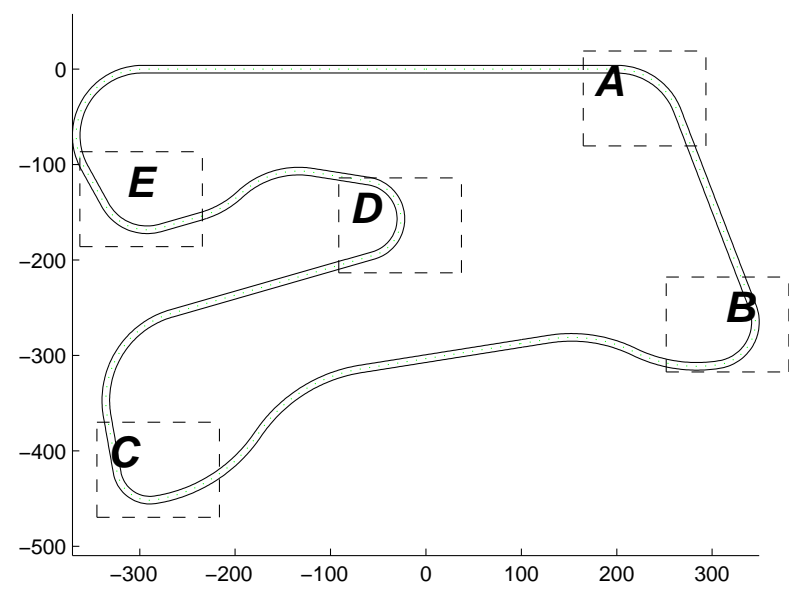

Fig. 15. Digitized map of Hockenheim Racing Circuit including five curves where AEC was triggered.

the MHA controller using the internal tyre model (black); (c) the resulting vehicle CG acceleration $\mathbf{a}^{\text {act }}$ from CarMaker (blue). Differences between $\mathbf{a}^{d}$ and $\mathbf{a}^{c m d}$ arise due to actuator limitations, while differences between $\mathbf{a}^{c m d}$ and $\mathbf{a}^{a c t}$ result from simplifications in the vehicle dynamics and tyre model used within the controller.

Discrepancies are most obvious during the transient phase following the initial control application, with a phase lag evident. However the three arrows converge well after the transition to more steady-state conditions. The key comparison is between $\mathbf{a}^{d}$ and $\mathbf{a}^{a c t}$ - if these vectors coincide, then bounded off-tracking is guaranteed by the above theory. During the AEC events, maximum off-tracking stays below $1 \mathrm{~m}$; though slightly in excess of the $0.8 \mathrm{~m}$ design target, some additional error is expected, given the phase lag between $\mathbf{a}^{a c t}$ and $\mathbf{a}^{d}$. Note that the acceleration vectors are approximately fixed in inertial coordinates, even though the reference is updated at each time-step, confirming the results of Section IV-C.

In Figure 18, the lateral deviation from the track centre is shown for the entire circuit, and the five interventions are highlighted with bold marking. While the unaided driver drifts

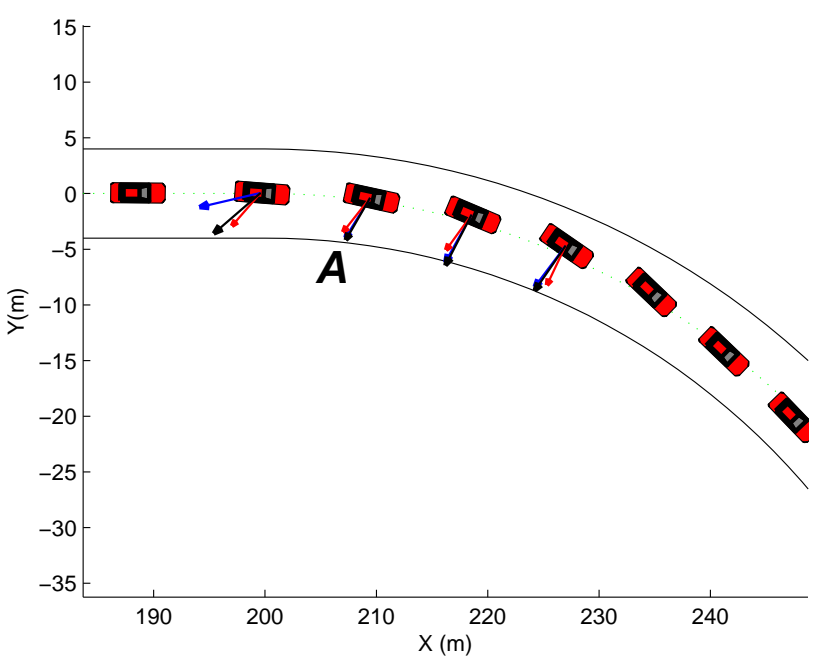

Fig. 16. Vehicle path and accelerations at corner $A$. Red arrows: acceleration targets; black arrows: MHA commanded accelerations; blue arrows: 'actual' accelerations from CarMaker.

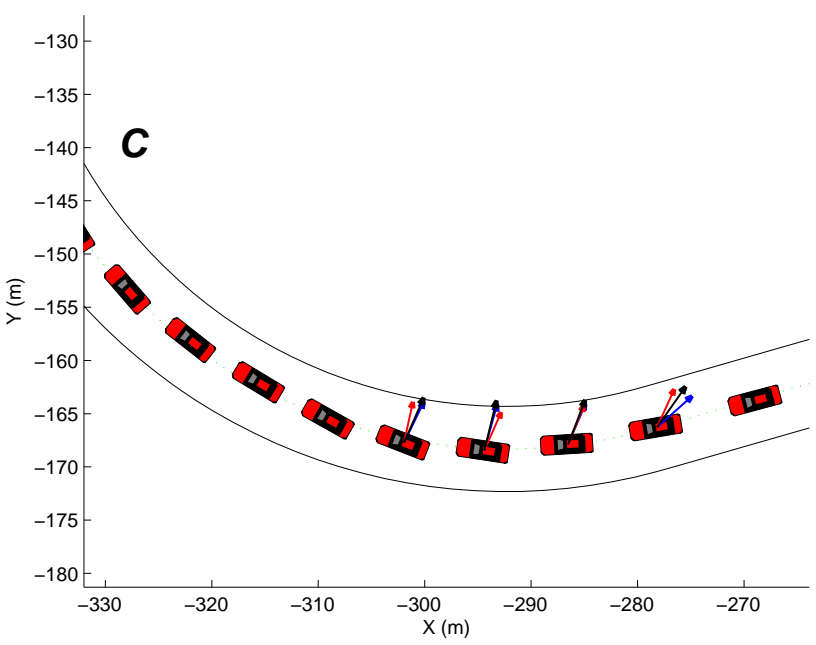

Fig. 17. Vehicle path and accelerations at corner $C$. Red arrows: acceleration targets; black arrows: MHA commanded accelerations; blue arrows: 'actual' accelerations from CarMaker.

up to $6 \mathrm{~m}$ from the centre-line, AEC limits the maximum to 1 $\mathrm{m}$.

After the second intervention, at around $s=500 \mathrm{~m}$, offtracking does reach $2 \mathrm{~m}$. This results from driver model limitations: after AEC control is released, aggressive acceleration from the PID speed control reduces the performance of steering control. Other, less extreme, deviations under driver model control are also evident.

We briefly consider vehicle lateral stability in the form of body side-slip angle - Figure 19. MHA is designed to limit excessive body side-slip [24] and in these simulations the upper bound was set at $8 \mathrm{deg}$, and this is a tunable parameter. This is approximately achieved and is slightly better than the peak values for the simulation without AEC. As with the target 
bound for off-tracking, there is some additional deviation due to transients, but overall lateral stability is maintained. Tighter control of body side-slip is possible, but if the upper bound is too small it will restrict the lateral forces at the rear axle and hence reduce acceleration tracking performance.

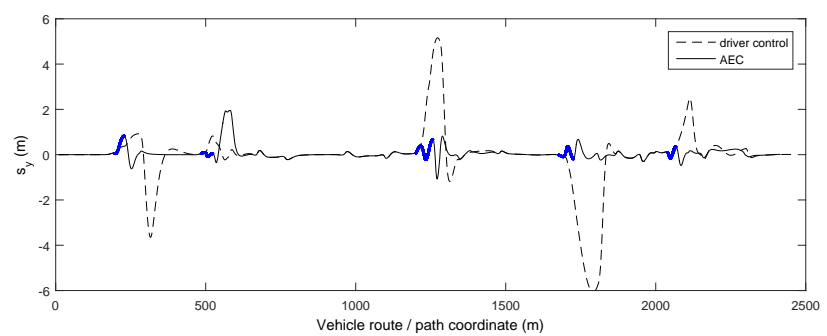

Fig. 18. Vehicle path plots in track coordinates. Bold markers indicates times when AEC is active.

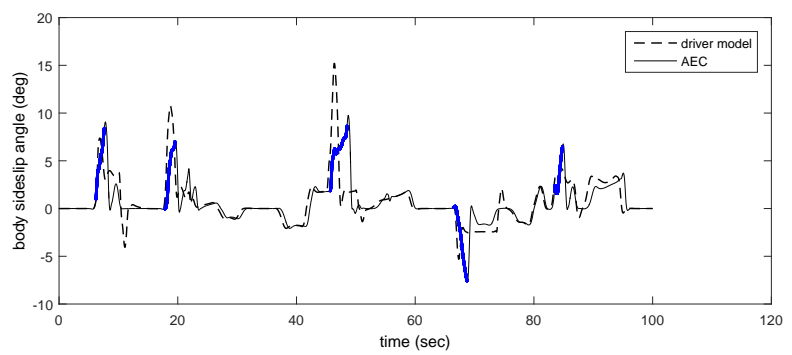

Fig. 19. Vehicle body side-slip angle. Bold line style indicates system interventions.

Figure 20 shows the front axle steering angle, with red dots highlighting the five interventions. Figure 21 shows the direct yaw moment contribution from the brake actuators (red), which provides roughly $50 \%$ of the total yaw moment (shown in black). Some brake torque 'chatter' is seen towards the end of some of the events.

\section{B. Highway Entry Curve with Reduced Friction}

Here the scenario is a highway entry road with low surface friction $\left(\mu_{s}=0.4\right)$ shown in Figure 22. The vehicle approaches from the right and eventually merges onto the highway. To represent the case where the driver over-estimates the road friction, $v_{\text {lim }}$ used by the driver model is set with $\mu_{p}=$ 0.42 while AEC is presumed aware of the lower friction with $\mu_{s}=0.4$ in the tyre model. Further, a conservative particle friction $\mu_{c}=0.35$ is used within the AEC algorithm. The vehicle path is shown Figure 23. With AEC enabled, maximum off-tracking is limited to around $1.5 \mathrm{~m}$. The acceleration vectors show slightly greater overall dispersion than previously, explaining the slightly greater off-tracking. It can be seen that the uncontrolled vehicle leaves the road (and the simulation stops). Note that the vehicle acceleration vector (blue arrow) has reduced magnitude compared with the expected acceleration (black arrow) and again there are transient delays apparent. There are seen to be three sub-interventions on the single curve, due to the tightening curvature and the driver model increasing speed whenever AEC is switched off.

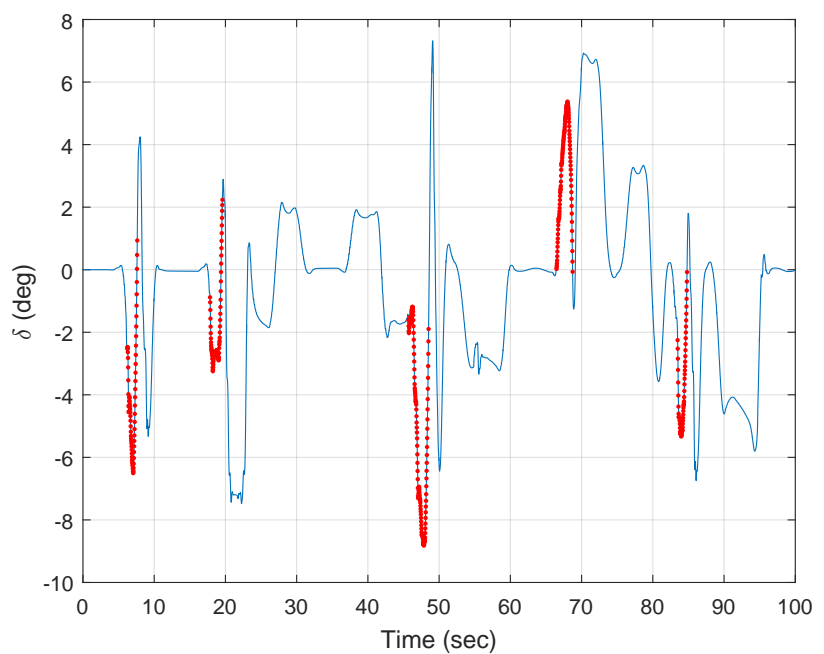

Fig. 20. Steering inputs at the front axle. AEC-controlled steering inputs are shown as red dots.

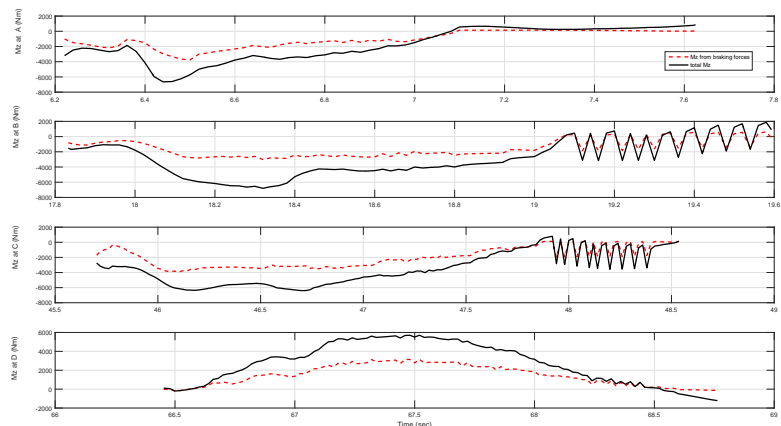

Fig. 21. Yaw moments due to MHA action at curves A to D. The direct yaw moment from braking is shown in red dashed line, comprising roughly $50 \%$ of the total, shown in black.

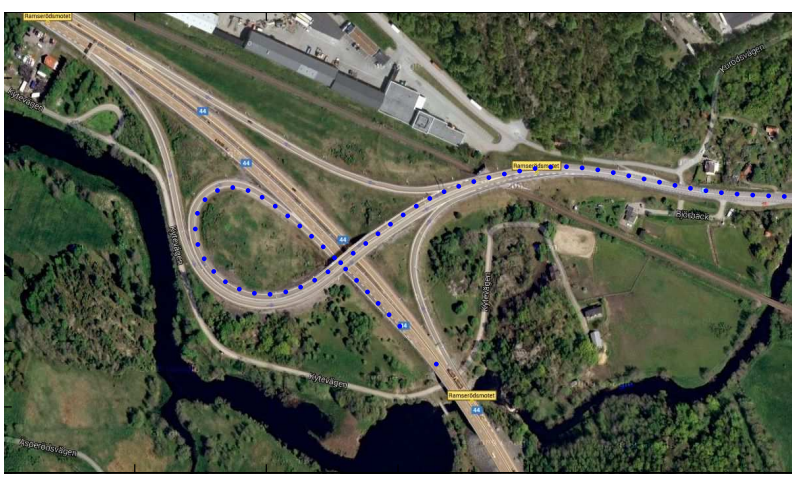

Fig. 22. Highway entry curve Råsserödsmotet, Uddevalla, Sweden; map of selected road geometry with fitted track model (courtesy: Google Maps, lat.58.3506, long.11.9797)

Here the various control system parameters were left unchanged from the previous scenario, i.e. there was no re-tuning of the MHA parameters other than to revise the friction estimates. The MHA internal tyre model was re-scaled according to the new value of $\mu_{s}$, and no further tyre model fitting was carried out. Example tyre forces are shown in Figure 24, here for the first AEC intervention. At the front-left wheel, $F_{x}^{t}$ 


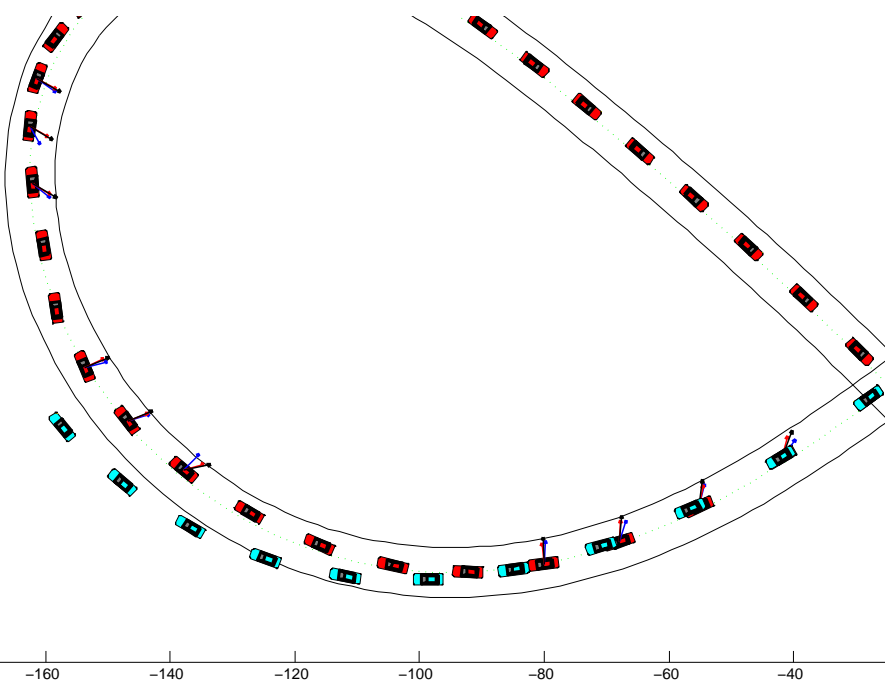

Fig. 23. Vehicle path on a highway entry curve; blue vehicle - no AEC system intervention; red vehicle - AEC system eneabled. Arrow labels: see Figure 16 caption.

follows the control input slightly better than for $F_{y}^{t}$, while for the rear-right tyre both force components are tracked well.
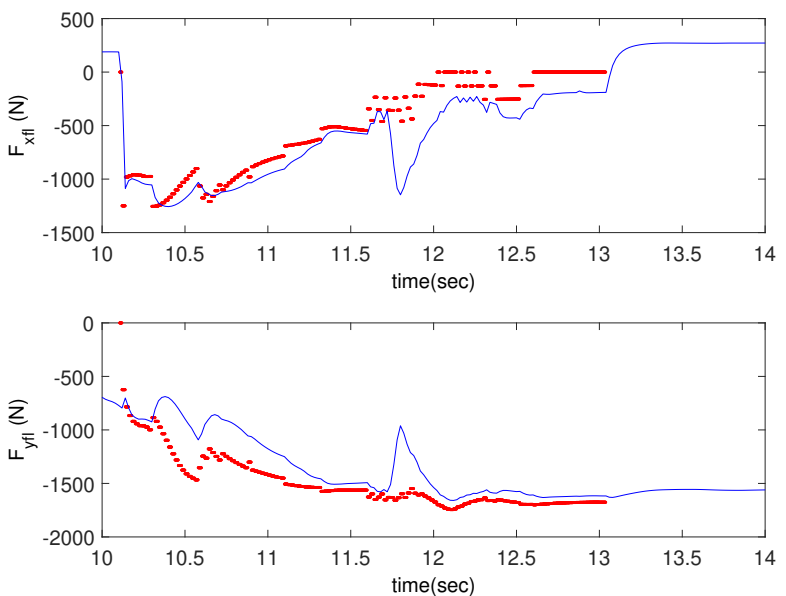

Fig. 24. Longitudinal and lateral tyre force control at the front-left tyre on the highway entry curve. The blue solid lines represents the 'actual' tyre forces (CarMaker output). The red dotted lines are the commanded forces from MHA.

\section{CONCLUSION}

This paper has presented theory, algorithms and simulations to formulate and test a concept for a future Automated Emergency Cornering (AEC) system. The proposed AEC system uses a digital map and vehicle kinematic data to trigger and update the motion reference. It also requires friction estimation to perform in a near-optimal way. It has a hierarchical structure, the upper level based on an optimal particle model, the lower level distributing control objectives via the MHA method. MHA has been shown capable of real-time operation and is equally applicable to high and low friction surfaces.
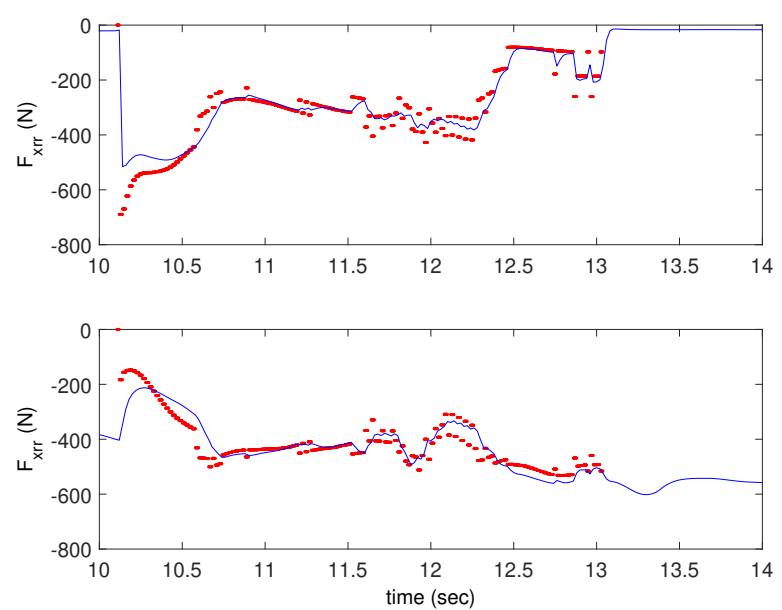

Fig. 25. Longitudinal and lateral tyre force control at the rear-right tyre (as for Figure 24).

In simulation, the proposed AEC system successfully prevented road departures, analogous to the way Autonomous Emergency Braking (AEB) systems anticipate and mitigate frontal collisions. AEC intervention was triggered at the point when further delay or sub-optimal response will necessarily lead to excessive lane departure. Because the system uses all available actuators and all available friction, a human driver could not be expected to achieve similar off-tracking performance, except by intervening earlier. Hence AEC is not expected to interfere with normal driving.

While simplified models are used for theoretical development and within in the controller, the high-fidelity vehicle dynamics software CarMaker was used to test the ability of AEC to achieve its purpose. The AEC system compares well to the predictions from the friction-limited particle on the high friction tests. On the lower friction there are longer transient delays is building the required forces, and some compensation may be necessary in terms of trigger timing. While refinements will be needed for robust implementation, this paper has defined the essential building blocks of a feasible AEC system.

\section{APPENDIX A \\ LATERAL VELOCITY REVERSAL}

This appendix verifies the existence of $e_{2}$ for the particle motion under constant acceleration $\mathbf{a}_{P}$ given in Eqn. 39, assuming the conditions of Section IV-C:

1) $e_{1}>0$ exists such that $v_{\perp}\left(e_{1}\right)>0$ (scenario assumption)

2) $v_{0}>v_{\text {lim }}\left(s_{0}\right)$ (over-speed condition)

3) $c(e) \geq 0$ for $e \geq e_{1}$ (unidirectional curvature)

The scenario of Figure 11 is drawn again in Figure 26, now with initial point $S$ corresponding to $e=e_{1}$ and therefore $v_{\perp}(S)>0$. Inertial coordinates $(X, Y)$ are selected at this point, with the $X$-axis parallel to the track tangent. The dashed curve $S P^{\prime}$ follows the path of the track but may be laterally offset at a constant distance from the track centre-line. Here $u$ denotes the longitudinal distance from $S$ along the curve; because of lateral offset, it differs slightly from the centre-line distance $e-e_{1}$, but is more convenient for this analysis. 


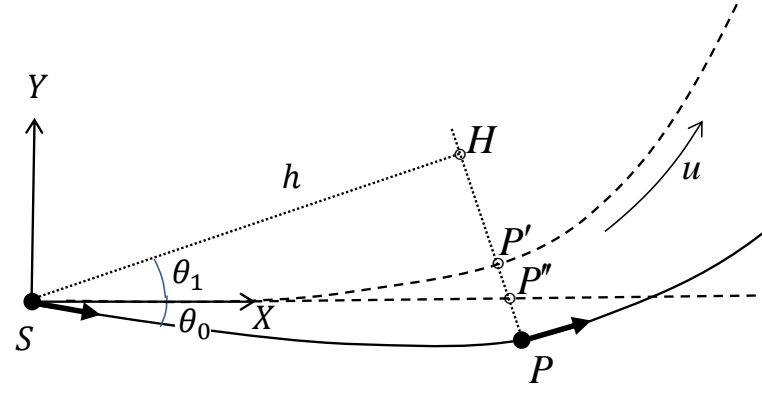

Fig. 26. Particle motion from $S$ to $P$ along a path of positive curvature. $\theta=\theta_{0}+\theta_{1}$ is the angle given in Eqn. 56.

For preview point $P$, the track-normal is drawn to define points $P^{\prime}, P^{\prime \prime}$, as shown; line $S H$ is parallel to the track tangent and is hence normal to line $P H$. Angle $\theta=\theta_{0}+\theta_{1}$ is the angle between the current path and the normal at the preview point. Condition $c(e) \geq 0$ implies $\theta_{1}$ is a nondecreasing function of $u$, while $v_{\perp}(S)>0$ implies $\theta_{0}>0$.

The requirement is to show $v_{\perp}(e)<0$ for some $e>e_{1}$. Equivalently, the bracketed expression in Eqn. 44 should become negative for some $u>0$ :

$$
\sin 2 \theta-\frac{h}{L}<0
$$

Since the stopping distance $L$ is a positive constant, and $h \geq 0$ for all possible road geometries, Eqn. 56 is immediately satisfied if $\sin 2 \theta<0$, i.e. if $\theta>\pi / 2$ for a sufficiently large distance along the track.

Hence we can limit attention to the case where the road does not turn through a full $90^{\circ}$ within the assumed preview horizon. Then we naturally expect $h$ to increase without any upper limit and this is now shown.

$$
\theta_{1} \leq \pi / 2-\theta_{0} .
$$

and hence $\cos \theta_{1}$ is bounded below

$$
\cos \theta_{1} \geq \sin \theta_{0}>0
$$

In Figure 26, let $x$ be the $X$-coordinate of point $P^{\prime}$. Since the tangent at $P^{\prime}$ is parallel to line $S H$,

$$
\frac{d x}{d y}=\cos \theta_{1}
$$

Hence, using Eqn. 58,

$$
x(u)=\int \cos \theta_{1} d u \geq \int \sin \theta_{0} d u=u \sin \theta_{0}
$$

Also, it is clear that distance $\left|S P^{\prime \prime}\right|=h / \cos \theta_{1}$ is greater than $x$, hence

$$
h \geq x \cos \theta_{1} \geq x \sin \theta_{0} \geq u \sin ^{2} \theta_{0} .
$$

\begin{tabular}{|c|c|c|c|}
\hline Parameter & $\begin{array}{l}\text { Physical } \\
\text { Meaning }\end{array}$ & Unit & Value \\
\hline$m$ & total mass & $\mathrm{kg}$ & 1174 \\
\hline$m_{s}$ & sprung mass & $\mathrm{kg}$ & 1020 \\
\hline$m_{u}$ & unsprung mass & $\mathrm{kg}$ & 154 \\
\hline$I_{z z}$ & $\begin{array}{l}\text { yaw moment of } \\
\text { inertia }\end{array}$ & $\mathrm{kgm}^{2}$ & 1360 \\
\hline$I_{x x}$ & $\begin{array}{l}\text { roll moment of } \\
\text { inertia }\end{array}$ & $\mathrm{kgm}^{2}$ & 350 \\
\hline$I_{y y}$ & $\begin{array}{l}\text { pitch moment of } \\
\text { inertia }\end{array}$ & $\mathrm{kgm}^{2}$ & 1290 \\
\hline$l_{f}$ & CG to front axle & $\mathrm{m}$ & 1.043 \\
\hline$l_{r}$ & CG to rear axle & $\mathrm{m}$ & 1.637 \\
\hline$w$ & track width & $\mathrm{m}$ & 1.530 \\
\hline$h$ & mass centre hight & $\mathrm{m}$ & 0.605 \\
\hline$\mu_{p}$ & $\begin{array}{l}\text { particle friction } \\
\text { coefficient }\end{array}$ & - & 0.8 \\
\hline$\mu_{c}$ & $\begin{array}{l}\text { AEC-estimated } \\
\text { friction }\end{array}$ & - & 0.8 \\
\hline$\mu_{s}$ & $\begin{array}{l}\text { surface friction } \\
\text { of the tyre model }\end{array}$ & - & 1.0 \\
\hline$R_{\mathrm{w}}$ & $\begin{array}{l}\text { loaded tyre ra- } \\
\text { dius }\end{array}$ & $\mathrm{m}$ & 0.3 \\
\hline$I_{\mathrm{W}}$ & $\begin{array}{l}\text { nominal wheel } \\
\text { rotational inertia }\end{array}$ & $\mathrm{kgm}^{2}$ & 0.5 \\
\hline$\Lambda$ & $\begin{array}{l}\text { front and rear } \\
\text { suspension ratio }\end{array}$ & - & 0.5 \\
\hline$\rho$ & air mass density & $\mathrm{kgm}^{3}$ & 1.2 \\
\hline$C_{d}$ & $\begin{array}{l}\text { air drag coeffi- } \\
\text { cient }\end{array}$ & - & 0.3 \\
\hline$A_{F}$ & $\begin{array}{l}\text { front area of the } \\
\text { vehicle }\end{array}$ & $\mathrm{m}^{2}$ & 2.4 \\
\hline$\tau$ & $\begin{array}{l}\text { brake/drive } \\
\text { torque } \\
\text { constant }\end{array}$ & $\mathrm{s}$ & 0.05 \\
\hline$B, C, D, E$ & $\begin{array}{l}\text { MF tyre coeffi- } \\
\text { cients }\end{array}$ & - & $0.7094,1.4097,1.0,0$ \\
\hline$i_{S}$ & $\begin{array}{l}\text { steering system } \\
\text { ratio }\end{array}$ & - & 17.0 \\
\hline
\end{tabular}

where Eqn. 60 has been used. Hence, if $u>L / \sin ^{2} \theta_{0}$, Eqn. 56 is again satisfied.

\section{APPENDIX B}

\section{VEHICLE PARAMETERS}

\section{REFERENCES}

[1] Lotta Jakobsson, Magdalena Lindman, Anders Axelson, Bengt Lokensgard, Mats Petersson, Bo Svanberg, and Jordanka Kovaceva. Addressing run off road safety. SAE International Journal of Passenger CarsMechanical Systems, 7(2014-01-0554):132-144, 2014.

[2] Cejun Liu and Rajesh Subramanian. Factors related to fatal singlevehicle run-off-road crashes. Technical report, 2009.

[3] Cejun Liu and Tony Jianqiang Ye. Run-off-road crashes: an on-scene perspective. Technical report, 2011.

[4] David LeBlanc. Road departure crash warning system field operational test: methodology and results. volume 1: technical report. 2006.

[5] John Vincent Bond III, Gerald H Engelman, Jonas Ekmark, Jonas LZ Jansson, M Nabeel Tarabishy, and Levasseur Tellis. Autonomous emergency braking system, February 25 2003. US Patent 6,523,912.

[6] Jessica B Cicchino. Effectiveness of forward collision warning systems with and without autonomous emergency braking in reducing policereported crash rates. Arlington, VA: Insurance Institute for Highway Safety, 2016.

[7] David J LeBlanc, Gregory E Johnson, Paul J Th Venhovens, Garth Gerber, Robert DeSonia, Robert D Ervin, Chiu-Feng Lin, A Galip Ulsoy, and Thomas E Pilutti. Capc: A road-departure prevention system. IEEE Control Systems Magazine, 16(6):61-71, 1996.

[8] Vassilios Morellas, Ted Morris, Lee Alexander, and Max Donath. Preview based control of a tractor trailer using dgps for preventing road departure accidents. In Proceedings of Conference on Intelligent Transportation Systems, pages 797-805. IEEE, 1997.

[9] Parag H Batavia, Dean A Pomerleau, and Charles E Thorpe. Predicting lane position for roadway departure prevention. In Proceedings of the IEEE intelligent vehicles symposium, pages 245-251, 1998.

[10] Cláudio Rosito Jung and Christian Roberto Kelber. Lane following and lane departure using a linear-parabolic model. Image and Vision Computing, 23(13):1192-1202, 2005. 
[11] A Kullack, I Ehrenpfordt, K Lemmer, and F Eggert. Reflektas: lane departure prevention system based on behavioural control. IET Intelligent Transport Systems, 2(4):285-293, 2008.

[12] Yasuhisa Hayakawa, Kou Sato, Yoshiaki Tabata, and Kenichi Egawa. Design of a lane departure prevention system with enhanced drivability. SAE International Journal of Passenger Cars-Mechanical Systems, 2(2009-01-0160):398-403, 2009.

[13] Nicoleta Minoiu Enache, Said Mammar, Sebastien Glaser, and Benoit Lusetti. Driver assistance system for lane departure avoidance by steering and differential braking. IFAC Proceedings Volumes, 43(7):471476, 2010.

[14] D Katzourakis, Mohsen Alirezaei, Joost CF de Winter, Matteo Corno, Riender Happee, A Ghaffari, and R Kazemi. Shared control for road departure prevention. In 2011 IEEE International Conference on Systems, Man, and Cybernetics, pages 1037-1043. IEEE, 2011.

[15] Abdelkader Merah, Kada Hartani, and Azeddine Draou. A new shared control for lane keeping and road departure prevention. Vehicle System Dynamics, 54(1):86-101, 2016.

[16] Dongkui Tan, Wuwei Chen, Hongbo Wang, and Zhengang Gao. Shared control for lane departure prevention based on the safe envelope of steering wheel angle. Control Engineering Practice, 64:15-26, 2017.

[17] Yiannis E Papelis, Ginger S Watson, and Timothy L Brown. An empirical study of the effectiveness of electronic stability control system in reducing loss of vehicle control. Accident Analysis \& Prevention, 42(3):929-934, 2010

[18] Sehyun Chang and Timothy J Gordon. Model-based predictive control of vehicle dynamics. International Journal of Vehicle Autonomous Systems, 5(1-2):3-27, 2007.

[19] Paolo Falcone, Francesco Borrelli, H Eric Tseng, Jahan Asgari, and Davor Hrovat. A hierarchical model predictive control framework for autonomous ground vehicles. In American Control Conference, 2008 pages 3719-3724. IEEE, 2008.

[20] Javad Ahmadi, Ali Khaki Sedigh, and Mansour Kabganian. Adaptive vehicle lateral-plane motion control using optimal tire friction forces with saturation limits consideration. IEEE Transactions on vehicular technology, 58(8):4098-4107, 2009.

[21] Derong Yang, Timothy J Gordon, Bengt Jacobson, and Mats Jonasson. Quasi-linear optimal path controller applied to post impact vehicle dynamics. IEEE transactions on intelligent transportation systems, 13(4):1586-1598, 2012

[22] Matthijs Klomp, Mathias Lidberg, and Timothy J Gordon. On optimal recovery from terminal understeer. Proceedings of the Institution of Mechanical Engineers, Part D: Journal of Automobile Engineering, 228(4):412-425, 2014.

[23] Derong Yang, Bengt Jacobson, Mats Jonasson, and Tim J Gordon. Closed-loop controller for post-impact vehicle dynamics using individual wheel braking and front axle steering. International Journal of Vehicle Autonomous Systems, 12(2):158-179, 2014.

[24] Yangyan Gao, Mathias Lidberg, and Timothy Gordon. Modified hamiltonian algorithm for optimal lane change with application to collision avoidance. MM Science Journal MAR, pages 576-584, 2015.

[25] John K Subosits and J Christian Gerdes. A synthetic input approach to slip angle based steering control for autonomous vehicles. In 2017 American Control Conference (ACC), pages 2297-2302. IEEE, 2017.

[26] Hongyan Guo, Feng Liu, Fang Xu, Hong Chen, Dongpu Cao, and Yan Ji. Nonlinear model predictive lateral stability control of active chassis for intelligent vehicles and its fpga implementation. IEEE Transactions on Systems, Man, and Cybernetics: Systems, 2017.

[27] Vincent A Laurense, Jonathan Y Goh, and J Christian Gerdes. Pathtracking for autonomous vehicles at the limit of friction. In 2017 American Control Conference (ACC), pages 5586-5591. IEEE, 2017.

[28] Y Gao. Vehicle Motion and Stability Control at the Limits of Handling via the Modified Hamiltonian Algorithm. $\mathrm{PhD}$ thesis, University of Lincoln, 2018

[29] Mohammadhossein Malmir, Marco Baur, and Luca Bascetta. A model predictive controller for minimum time cornering. In 2018 International Conference of Electrical and Electronic Technologies for Automotive, pages 1-6. IEEE, 2018.

[30] Victor Fors, Björn Olofsson, and Lars Nielsen. Slip-angle feedback control for autonomous safety-critical maneuvers at-the-limit of friction. In International Symposium on Advanced Vehicle Control (AVEC), 2018.

[31] Joseph Funke, Matthew Brown, Stephen M Erlien, and J Christian Gerdes. Collision avoidance and stabilization for autonomous vehicles in emergency scenarios. IEEE Transactions on Control Systems Technology, 25(4):1204-1216, 2017.
[32] András Mihály, Balázs Németh, and Péter Gáspár. Look-ahead control of road vehicles for safety and economy purposes. In 2014 European Control Conference (ECC), pages 714-719. IEEE, 2014.

[33] Yangyan Gao, Timothy Gordon, and Mathias Lidberg. A flexible control allocation method for terminal understeer mitigation. In Students on Applied Engineering (ICSAE), International Conference for, pages 18 23. IEEE, 2016.

[34] Yangyan Gao, Timothy Gordon, and Mathias Lidberg. Implementation of a modified hamiltonian algorithm for control allocation. In Advanced Vehicle Control: Proceedings of the 13th International Symposium on Advanced Vehicle Control (AVEC'16), September 13-16, 2016, Munich, Germany, page 157. CRC Press, 2016.

[35] IPG CarMaker. Users guide version 4.5. 2. IPG Automotive, Karlsruhe, Germany, 2014

[36] Hans B Pacejka and Egbert Bakker. The magic formula tyre model. Vehicle system dynamics, 21(S1):1-18, 1992

[37] William F Milliken, Douglas L Milliken, et al. Race car vehicle dynamics, volume 400. Society of Automotive Engineers Warrendale, 1995.

[38] Hans B Pacejka. Tyre and vehicle dynamics, p 511-562. Delft University of Technology, 2002.

[39] TJ Gordon and Matt C Best. On the synthesis of driver inputs for the simulation of closed-loop handling manoeuvres. International Journal of Vehicle Design, 40(1-3):52-76, 2005.

[40] IPG CarMaker. Ipg driver user manual 6.4. IPG Automotive, Karlsruhe, Germany, 2014

[41] Ola HäRkegåRd and S Torkel Glad. Resolving actuator redundancyoptimal control vs. control allocation. Automatica, 41(1):137-144, 2005.

[42] Johannes Tjonnas and Tor A Johansen. Stabilization of automotive vehicles using active steering and adaptive brake control allocation. IEEE Transactions on Control Systems Technology, 18(3):545-558, 2010.

[43] Tor A Johansen and Thor I Fossen. Control allocationa survey. Automatica, 49(5):1087-1103, 2013

[44] Hans P Geering. Optimal control with engineering applications. Springer, 2007.

[45] Arthur Earl Bryson. Applied optimal control: optimization, estimation and control. Routledge, 2018.

[46] Ignatius Jozef Maria Besselink. Shimmy of aircraft main landing gears. na, 2000.

[47] Uwe Kiencke and Lars Nielsen. Automotive control systems: for engine, driveline, and vehicle, 2000. 